\title{
Seismic interferometry using multidimensional deconvolution and crosscorrelation for crosswell seismic reflection data without borehole sources
}

\author{
$\operatorname{AUTHOR}(\mathrm{S})$ : \\ Minato, Shohei; Matsuoka, Toshifumi; Tsuji, \\ Takeshi; Draganov, Deyan; Hunziker, Jurg; \\ Wapenaar, Kees
}

\section{CITATION:}

Minato, Shohei ... [et al]. Seismic interferometry using multidimensional deconvolution and crosscorrelation for crosswell seismic reflection data without borehole sources. GEOPHYSICS 2011, 76(1): SA19_SA34

\section{ISSUE DATE:}

2011-01

URL:

http://hdl.handle.net/2433/163456

RIGHT:

(C)2011 Society of Exploration Geophysicists 


\title{
Seismic interferometry using multidimensional deconvolution and crosscorrelation for crosswell seismic reflection data without borehole sources
}

\author{
Shohei Minato ${ }^{1}$, Toshifumi Matsuoka ${ }^{1}$, Takeshi Tsuji ${ }^{1}$, Deyan Draganov², Jürg Hunziker ${ }^{2}$, and \\ Kees Wapenaar ${ }^{2}$
}

\begin{abstract}
Crosswell reflection method is a high-resolution seismic imaging method that uses recordings between boreholes. The need for downhole sources is a restrictive factor in its application, for example, to time-lapse surveys. An alternative is to use surface sources in combination with seismic interferometry. Seismic interferometry (SI) could retrieve the reflection response at one of the boreholes as if from a source inside the other borehole. We investigate the applicability of SI for the retrieval of the reflection response between two boreholes using numerically modeled field data. We compare two SI approaches - crosscorrelation (CC) and multidimensional deconvolution (MDD). SI by MDD is less sensitive to underillumination from the source distribution, but requires inversion of the recordings at one of the receiver arrays from all the available sources. We find that the inversion problem is illposed, and propose to stabilize it using singular-value decomposition. The results show that the reflections from deep boundaries are retrieved very well using both the $\mathrm{CC}$ and MDD methods. Furthermore, the MDD results exhibit more realistic amplitudes than those from the $\mathrm{CC}$ method for downgoing reflections from shallow boundaries. We find that the results retrieved from the application of both methods to field data agree well with crosswell seismic-reflection data using borehole sources and with the logged P-wave velocity.
\end{abstract}

\section{INTRODUCTION}

Seismic interferometry (SI) can be used to retrieve pseudoseismic data between receivers. Typically this is achieved by crosscorrelating observed wavefields.
The concept of SI finds its origin in a paper from Claerbout (1968). He showed that for a horizontally layered earth, the autocorrelation of passive seismic data from a buried plane-wave source produces a plane-wave reflection response at the free surface.

Since the work of Claerbout, the understanding of this technique has grown remarkably. In the exploration community, Schuster (2001) and Schuster et al. (2004) show that SI can be applicable not only for buried noise sources, but for controlled sources. Snieder (2004) discusses the details of SI by the stationary phase method for a scattering medium. Wapenaar et al. (2002) and Wapenaar (2004) use reciprocity theorems to prove that the crosscorrelation and summation yield the Green's function in 3D inhomogeneous media. Curtis et al. (2006), Larose et al. (2006), and Schuster (2009) give recent overviews of different applications of SI in both exploration and global seismology.

An important advantage of the application of interferometry to seismic exploration is that it allows flexibility of source and receiver configurations. To use SI for exploration, seismic receivers are placed in the vicinity of the exploration target and the wavefields generated by artificial or naturally occurring seismic sources are recorded. When these recorded data are crosscorrelated, one obtains new recordings at the receivers as if originating from one of the receivers that was turned into a virtual source.

SI with naturally occurring noise sources has become a standard technique in global and regional seismology. For example, Shapiro et al. (2005) retrieve surface waves from ambient seismic noise recorded by the USArray network in California and estimate their group velocity. In exploration seismology, where reflection information is desired, application of SI with noise sources has proven more difficult. Draganov et al. (2009) retrieved reflection arrivals from ambient seismic noise in Libya, and used the retrieved reflections to obtain a pseudo-3D image of the subsurface.

SI with active sources has gained much popularity in exploration

Manuscript received by the Editor 12 April 2010; revised manuscript received 9 July 2010; published online 10 January 2011; corrected version published online 11 January 2011.

${ }^{1}$ Kyoto University, Department of Urban Management, Kyoto, Japan. E-mail: s_minato@earth.kumst.kyoto-u.ac.jp; matsuoka@earth.kumst.kyoto-u.ac.jp; tsuji@earth.kumst.kyoto-u.ac.jp.

${ }^{2}$ Delft University of Technology, Department of Geotechnology, Delft, The Netherlands. E-mail: d.s.draganov@tudelft.nl; j.w.hunziker@tudelft.nl; c.p.a.wapenaar@tudelft.nl.

(C) 2011 Society of Exploration Geophysicists. All rights reserved. 
seismology. An example is the application for the retrieval of direct or scattered surface waves for their subsequent elimination from controlled-source reflection records (Dong et al., 2006; Halliday et al., 2007, 2010). As another example, applications to borehole seismic exploration methods, such as vertical seismic profiling (VSP), are studied (Jiang, 2006; Yu and Schuster, 2006). Furthermore, Bakulin and Calvert (2006) develop a technique to obtain virtual wavefields whose virtual sources are inside a single deviated borehole by crosscorrelating wavefield observations from controlled sources on the ground surface. They show that this technique removes the effect of the overburden, for example, scattering noise generated in weathered layers. It has been shown that by using this approach, boundaries of salt domes can be imaged (Willis et al., 2006; Xiao et al., 2006; Hornby and Yu, 2007), and structures underlying complex overburden can be imaged with higher resolution (Bakulin and Calvert, 2006). Mehta et al. (2007) show that the results from this technique can be improved if the crosscorrelation is applied to separated upgoing and downgoing fields.

SI has been applied to crosswell seismic data (Torii et al., 2006; Minato et al., 2007; Mehta et al., 2008a, 2010). The conventional crosswell seismic-reflection method can produce high-resolution images of subsurface structures between two boreholes when the seismic sources and receivers are installed in the boreholes (e.g., Harris et al., 1995). The advantages of crosswell seismic surveys over surface seismic surveys are that they provide higher resolution of the target structures, the data are free of surface waves, and the results are less affected by shallow, low-velocity-weathered layers. However, conventional crosswell surveys would require downhole sources, such as special vibrators that do not damage the borehole walls, and because these borehole sources produce less energy, the width of the survey spread is restricted. SI allows subsurface structures to be imaged from two boreholes without using borehole sources.

Minato et al. (2007) place receiver arrays in two vertical boreholes and use observations of the wavefield generated by surface energy sources to image subsurface structures. In that study, they consider only active sources on the ground surface, as used for conventional VSP surveys. By installing surface sources, the recorded wavefields in the boreholes are dominated by the upgoing reflections which usually are used for VSP reflection imaging (Oristaglio, 1985). In the following, we use surface sources because evaluation of the application of crosswell interferometry is simpler if controlled surface sources with known physical properties are used. The use of surface energy sources gives us operational advantages over conventional crosswell surveys. In particular, this method significantly reduces the cost of time-lapse crosswell surveys. The restriction of spread width (distance between boreholes) for the conventional crosswell reflection method using downhole sources can be overcome by using high-energy sources at the surface.

There is a large range of potential applications of this technique for long-distance crosswell reflection surveys over vast areas. Nevertheless, using surface sources would bring inherent limitations, as discussed above, because of the extra propagation of the surface signals to the observation boreholes, especially when a strongly weathered layer is present.

Underlying assumptions for the retrieval of responses between two receivers using crosscorrelation are those of a lossless medium and a homogeneous illumination of the receivers. To overcome these restrictions, it was proposed to use crossconvolution instead of crosscorrelation (Slob et al., 2007; Halliday and Curtis, 2009) or trace deconvolution (Vasconcelos and Snieder, 2008a, 2008b).

Wapenaar et al. (2008b) propose a new approach to seismic interferometry that is based on multidimensional deconvolution (MDD) instead of the conventional crosscorrelation (CC). Wapenaar et al. (2008a) demonstrate that this method is useful for application to electromagnetic data. The differences between the CC and MDD methods are: (1) MDD is valid in dissipative media, (2) MDD compensates for the source spectrum, (3) MDD could compensate for irregular source arrays, and (4) MDD accounts for the effects of onesided illumination. Because MDD compensates for the spectrum of the source wavelets, it could result in higher-resolution images than the CC method. Even though the results from the CC method normally are deconvolved for the source spectra after correlation and summation, this deconvolution might not be trivial. Furthermore, when source arrays are irregular, wave amplitudes and traveltimes retrieved by the MDD method better represent the true wavefield than those from the $\mathrm{CC}$ method: The amplitudes of the wavefield retrieved by the $\mathrm{CC}$ method differ from the true wavefield, and the integration of the crosscorrelation results from the irregular source array would result in lower signal-to-noise ratio of the retrieved results (e.g., Snieder et al., 2006; Mehta et al., 2008b; Wapenaar et al., 2008b). On the other hand, the MDD method has several constraints: (1) MDD requires receiver arrays and therefore cannot be applied to a single receiver configuration, (2) MDD tends to be more CPU intensive than the CC method by array operation, and (3) MDD possibly is unstable because the inversion problem may be ill-conditioned.

Here, we describe the theory of crosswell seismic interferometry by CC and MDD. This is the first application of MDD for crosswell seismic data. We introduce singular-value decomposition (SVD) to stabilize the MDD solution because the source-receiver configuration introduces an ill-posed problem for solving the MDD relationship.

Introducing SVD inversion for MDD is a different approach from the damped-least-squares inversion for electromagnetic data (Wapenaar et al., 2008a) and that for seismic data with a surface receiver array (Wapenaar et al., 2008b). We apply the methods to both numerically modeled and field data. The reflection profiles that we retrieve show that the imaged reflection boundaries from CC and MDD agree well with the velocity model.

\section{SEISMIC INTERFEROMETRY}

\section{Seismic interferometry by crosscorrelation}

The governing equation of SI by the $\mathrm{CC}$ method is derived from two-way wavefield reciprocity and the principle of time-reversal invariance (Wapenaar and Fokkema, 2006). It is described in the space-frequency domain as

$$
2 \Re\left\{\hat{G}\left(\mathbf{x}_{A}, \mathbf{x}_{B}, \omega\right)\right\} \approx \frac{2}{\rho c} \oint_{\partial \mathbb{D}} \hat{G}\left(\mathbf{x}_{A}, \mathbf{x}, \omega\right) \hat{G}^{*}\left(\mathbf{x}_{B}, \mathbf{x}, \omega\right) d^{2} \mathbf{x},
$$

where $\mathbf{x}=\left(x_{1}, x_{2}, x_{3}\right)$ is a position vector in Cartesian coordinates, $\hat{G}\left(\mathbf{x}_{A}, \mathbf{x}_{B}, \omega\right)$ represents the Green's function for a signal of angular frequency $\omega$ at receiver $\mathbf{x}_{A}$ from a source at $\mathbf{x}_{B}$ and $\hat{G}\left(\mathbf{x}_{B}, \mathbf{x}, \omega\right)$ represents the Green's function at receiver $\mathbf{x}_{B}$ from a monopole source at $\mathbf{x}$. The asterisk superscript indicates complex conjugation. Multiple sources $\mathbf{x}$ are distributed along an arbitrarily shaped, closed-surface 
$\partial \mathrm{D}$ and integration along this boundary is evaluated.

The multiplication of the complex-conjugated Green's function at $\mathbf{x}_{B}$ with the Green's function at $\mathbf{x}_{A}$ in the frequency domain corresponds to a crosscorrelation in the time domain. Therefore, $\hat{G}\left(\mathbf{x}_{A}, \mathbf{x}_{B}, \omega\right)$ can be retrieved by integrating crosscorrelations of the wavefield observed at receivers $\mathbf{x}_{A}$ and $\mathbf{x}_{B}$ along the closed-surface $\partial \mathrm{D}$ where sources exist.

Note that the interferometric relation 1 was obtained from an exact relation after several approximations. First, it was assumed that the medium along and outside $\partial \mathbb{D}$ was homogeneous. Second, a farfield approximation was applied to exchange dipole sources in the exact representation with monopole ones. In practical applications, these approximations might lead to (possibly significant) amplitude errors and spurious events.

For application of interferometry to crosswell seismic data, the energy sources that generate the wavefield can be either artificial active sources or natural passive sources. In this study, we consider only active sources on the ground surface, as used for conventional VSP surveys. The use of high-energy surface sources allows the crosswell reflection method to be used for widely separated boreholes. Furthermore, evaluation of the application of crosswell interferometry is simpler if controlled surface sources with known physical properties are used.

For our application of crosswell SI (equation 1), we use sources on the ground surface and receivers in the boreholes (Torii et al., 2006; Minato et al., 2007; Mehta et al., 2008a, 2010) as shown in Figure 1. Multiple surface sources are sequentially excited and the resultant wavefields are observed at all borehole receivers (Figure 1). Here, the Green's function on the left-hand side of equation 1 represents the virtual wavefield at well 1, assuming virtual sources at well 2; this is represented by $\hat{G}^{L}\left(\mathbf{x}_{A}, \mathbf{x}, \omega\right)$ in Figure 1 . The two Green's functions on the right-hand side of equation 1 represent the observed wavefields at the boreholes. However, in the field we do not measure the Green's function, but instead measure the pressure field. Therefore, the two Green's functions on the right-hand side of equation 1 are changed to pressure fields $\hat{p}$ including the Fourier transform of the wavelet $\hat{S}(\omega)$ of the surface sources (in field measurements this wavelet also should account for the instrument response of the receivers) as

$$
\begin{aligned}
& \hat{p}\left(\mathbf{x}_{A}, \mathbf{x}, \omega\right)=\hat{S}(\omega) \hat{G}\left(\mathbf{x}_{A}, \mathbf{x}, \omega\right), \\
& \hat{p}\left(\mathbf{x}_{B}, \mathbf{x}, \omega\right)=\hat{S}(\omega) \hat{G}\left(\mathbf{x}_{B}, \mathbf{x}, \omega\right) .
\end{aligned}
$$

Consequently, we retrieve a response that is convolved with the autocorrelation of the surface-source wavelet. Therefore, the Green's function on the left-hand side of equation 1 is exchanged for $\hat{C}$, representing a Green's function multiplied by the power spectrum of the surface-source wavelet (i.e., the Fourier transform of the autocorrelation):

$$
2 \Re\left\{\hat{C}\left(\mathbf{x}_{A}, \mathbf{x}_{B}, \omega\right)\right\} \approx \frac{2}{\rho c}|\hat{S}(\omega)|^{2} \oint_{\partial \mathrm{D}} \hat{G}\left(\mathbf{x}_{A}, \mathbf{x}, \omega\right) \hat{G}^{*}\left(\mathbf{x}_{B}, \mathbf{x}, \omega\right) d^{2} \mathbf{x}
$$

In our case of crosswell geometry, the integration boundary of equation 4 does not take the form of a closed surface because the source distribution is localized only on the earth's surface.

The computation procedure can be described as follows. The wavefield $\hat{C}\left(\mathbf{x}_{A}, \mathbf{x}, \omega\right)$, which propagates from one receiver in well 2 $(\mathbf{x})$ to another receiver in well $1\left(\mathbf{x}_{A}\right)$, can be retrieved from

$$
\hat{C}\left(\mathbf{x}_{A}, \mathbf{x}, \omega\right) \approx \sum_{k=1}^{N} \hat{p}\left(\mathbf{x}_{A}, \mathbf{x}_{S}^{(k)}, \omega\right) \hat{p}^{*}\left(\mathbf{x}, \mathbf{x}_{S}^{(k)}, \omega\right)
$$

where $\hat{p}^{*}\left(\mathbf{x}, \mathbf{x}_{S}^{(k)}, \omega\right)$ is the complex-conjugated wavefield observed at $\mathbf{x}$ from the $k$ th surface source, and $\hat{p}\left(\mathbf{x}_{A}, \mathbf{x}_{S}^{(k)}, \omega\right)$ is the wavefield at $\mathbf{x}_{A}$ from the $k$ th surface source. Note that we changed the notations in equation 1 from the source position $\mathbf{x}$ in the integral to $\mathbf{x}_{S}^{(k)}$, and the receiver position $\mathbf{x}_{B}$ to $\mathbf{x}$. Also, we skipped the factor $2 / \rho c$. To account for the integral of equation 4 , the correlation products are stacked over the interval of the surface-source array with the maximum number of elements $N$. In the examples that we are showing, we look only at the causal part of $\hat{C}$ in equation 5 because, as a result of the horizontally layered subsurface and ignoring backscattering, the acausal part of $\hat{C}$ would be retrieved from surface sources to the left of well 1 , which we do not have.

Equation 5 is similar to the virtual source method of Bakulin and Calvert (2006), but in their work, they implement the crosscorrelation of the gated direct field in $\mathbf{x}$ with the full wavefield at $\mathbf{x}_{A}$ to improve the retrieved upgoing events. Similarly, Mehta et al. (2007) show that correlation of separated downgoing waves in $\mathbf{x}$ with separated upgoing or downgoing waves at $\mathbf{x}_{A}$ improves the retrieval of events. Gating and/or separating wavefields before crosscorrelation can be regarded as an optional processing of equation 5, which is a crosscorrelation between total wavefields.

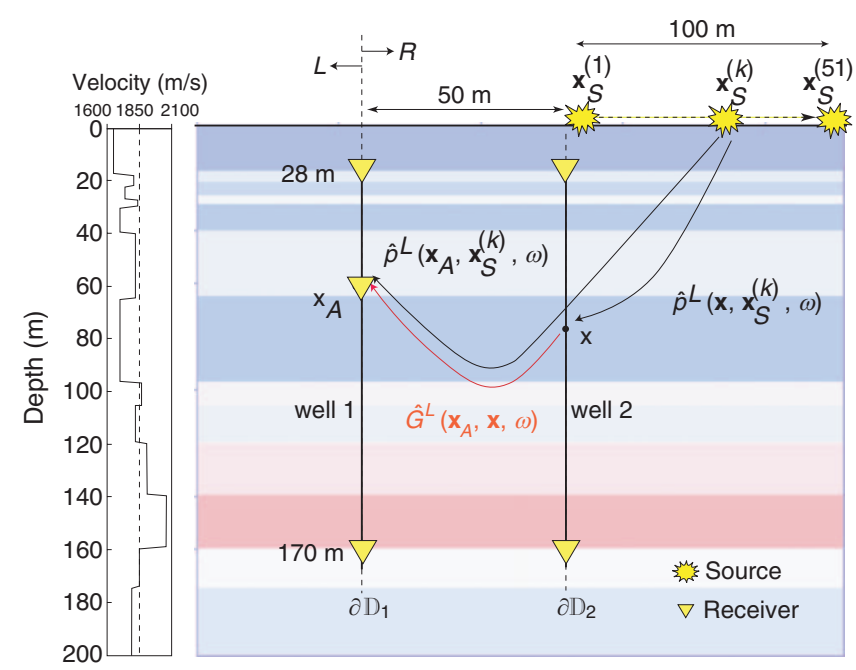

Figure 1. Velocity model and source-receiver configuration used for numerical modeling. There are 72 receivers at 2 -m intervals in each borehole and 51 sources $\left(\mathbf{x}_{S}^{(1)}\right.$ to $\left.\mathbf{x}_{S}^{(51)}\right)$ at the surface. The expressions $\mathbf{x}_{S}^{(k)}, \mathbf{x}$, and $\mathbf{x}_{A}$ represent the source and the two observation locations as used in equations 5 and 7 , whereas $\partial \mathrm{D}_{1}$ and $\partial \mathrm{D}_{2}$ indicate the chosen position of the boundaries from equation 6 . The expression $\hat{p}^{L}\left(\mathbf{x}_{A}, \mathbf{x}_{S}^{(k)}, \omega\right)$ is the acoustic wavefield observed at receiver position $\mathbf{x}_{A}$ in well 1 from a physical source at the surface at $\mathbf{x}_{S}^{(k)}$. The expression $\hat{p}^{L}\left(\mathbf{x}, \mathbf{x}_{S}^{(k)}, \omega\right)$ is the acoustic wavefield observed at receiver position $\mathbf{x}$ in well 2 from the same physical source at $\mathbf{x}_{S}^{(k)}$. The expression $\hat{G}^{L}\left(\mathbf{x}_{A}, \mathbf{x}, \omega\right)$ represents the crosswell Green's function at receiver position $\mathbf{x}_{A}$ in well 1, assuming that receiver $\mathbf{x}$ in well 2 is the virtual source position. 


\section{Seismic interferometry by multidimensional deconvolution}

Seismic interferometry by the MDD method is based on one-way reciprocity theory (Wapenaar et al., 2008a). One-way reciprocity refers to the relationship between wavefields or diffusion fields that can be separated into upgoing and downgoing components. In this study, we consider one-way reciprocity of scalar fields $\hat{p}^{+}$and $\hat{p}^{-}$, which represent downgoing and upgoing acoustic wavefields, respectively. The one-way reciprocity theorem of the convolution type is represented in the space-frequency domain as

$$
\int_{\partial \mathrm{D}_{2}}\left\{\hat{p}_{A}^{+} \hat{p}_{B}^{-}-\hat{p}_{A}^{-} \hat{p}_{B}^{+}\right\} d^{2} \mathbf{x}=\int_{\partial \mathrm{D}_{1}}\left\{\hat{p}_{A}^{+} \hat{p}_{B}^{-}-\hat{p}_{A}^{-} \hat{p}_{B}^{+}\right\} d^{2} \mathbf{x},
$$

where $\partial \mathrm{D}_{2}$ and $\partial \mathrm{D}_{1}$ represent horizontal boundaries of infinite extent $\left(\partial \mathrm{D}_{1}\right.$ lies below $\left.\partial \mathrm{D}_{2}\right)$. Subscripts $A$ and $B$ in equation 6 indicate two independent states, i.e., state $A$ and state $B$ could have different source positions and medium parameters. Two assumptions are required for equation 6: No sources should be located between the boundaries $\partial \mathbb{D}_{2}$ and $\partial \mathbb{D}_{1}$, and the physical parameters of the transmitting medium enclosed by the two boundaries should be identical for states $A$ and $B$. Substituting the observed wavefield from the actual source-receiver configuration into one of these states, and the desired wavefield (as the unknown function) for the virtual sourcereceiver configuration into the other state, leads to a relationship that is solved in the MDD method.

For the crosswell geometry, the derivation of the MDD relationship can be accomplished by imagining the configuration described above as rotated by $90^{\circ}$. In this situation, the upgoing and downgoing waves can be regarded as right-going and left-going waves, respectively. Furthermore, $\partial \mathrm{D}_{2}$ and $\partial \mathrm{D}_{1}$ in equation 6 become vertical boundaries. We then substitute the observed acoustic wavefield $\left(\hat{p}_{B}^{+}\right.$ and $\hat{p}_{B}^{-}$, or $\hat{p}_{B}^{L}$ and $\hat{p}_{B}^{R}$ in the new configuration) from the surface sources into state $B$ of equation 6 , and the acoustic wavefield $\left(\hat{p}_{A}^{+}\right.$and $\hat{p}_{A}^{-}$, or $\hat{p}_{A}^{L}$ and $\hat{p}_{A}^{R}$ in the new configuration) of the desired crosswell wavefield into state $A$. Here, we place two receiver borehole positions to correspond with the vertical boundaries $\partial \mathrm{D}_{2}$ and $\partial \mathrm{D}_{1}$ (Figure 1). Thus, we obtain the following convolution relationship between the observed wavefields from surface sources and the crosswell wavefield that we want to estimate (see Appendix A for details):

$$
\hat{p}^{L}\left(\mathbf{x}_{A}, \mathbf{x}_{S}, \omega\right)=\int_{\partial D_{2}} \hat{G}^{L}\left(\mathbf{x}_{A}, \mathbf{x}, \omega\right) \hat{p}^{L}\left(\mathbf{x}, \mathbf{x}_{S}, \omega\right) d^{2} \mathbf{x},
$$

where $\hat{p}^{L}\left(\mathbf{x}_{A}, \mathbf{x}_{S}, \omega\right)$ is the acoustic wavefield observed at receiver position $\mathbf{x}_{A}$ in well 1 from a physical source at the surface at $\mathbf{x}_{S}$. Similarly, $\hat{p}^{L}\left(\mathbf{x}, \mathbf{x}_{S}, \omega\right)$ is the acoustic wavefield observed at receiver position $\mathbf{x}$ in well 2 from the same physical source at $\mathbf{x}_{S}$ (see Figure 1). The superscript $L$ indicates decomposed leftward-propagating wavefields. The expression $\hat{G}^{L}\left(\mathbf{x}_{A}, \mathbf{x}, \omega\right)$ is the function to be retrieved and represents the crosswell Green's function at receiver position $\mathbf{x}_{A}$ in well 1 , assuming that receiver $\mathbf{x}$ in well 2 is the virtual source position. Note that $\partial \mathbb{D}_{2}$ of equation 7 is a $2 \mathrm{D}$ surface; however, in practice, our borehole represents a $1 \mathrm{D}$ boundary in the vertical direction. Therefore, we approximate $\partial \mathrm{D}_{2}$ as a $1 \mathrm{D}$ line in the vertical direction at the borehole positions.

MDD processing usually is done in the frequency domain, and an integral is evaluated along all receiver positions in well 2 (equation
7). Equation 7 states that integrating the product (in the frequency domain) of crosswell Green's functions and observed records at well 2 for all receiver positions $\left(\partial \mathrm{D}_{2}\right)$ gives the observed records at well 1. This relationship is exact (assuming a 2D configuration) when the following three conditions are satisfied: (1) $\partial \mathbb{D}_{2}$ (length of the borehole array at well 2) is of infinite extent, (2) the space to the left of well 1 is nonreflecting (i.e., no wavefields, such as reflections, originate from the left of well 1, and (3) the physical source $\mathbf{x}_{S}$ is located to the right of well 2 (see Appendix A for detail).

Contrary to the CC method (equation 5), MDD needs integration not over sources, but over receivers. This implies that the MDD method has the potential to be applied even for complex irregular source distributions.

Resolving $\hat{G}^{L}\left(\mathbf{x}_{A}, \mathbf{x}, \omega\right)$ from equation 7 requires MDD. If we consider one receiver position in well $1\left(\mathbf{x}_{A}\right)$ and multiple source positions $\left(\mathbf{x}_{S}\right)$, and if we replace the integral by a summation over the receivers of well $2\left(\partial \mathrm{D}_{2}\right)$, equation 7 can be written in a matrix-vector notation as

$$
\begin{aligned}
\left(\hat{p}^{L}\left(\mathbf{x}_{A}, \mathbf{x}_{S}^{(1)}\right) \ldots \hat{p}^{L}\left(\mathbf{x}_{A}, \mathbf{x}_{S}^{(N)}\right)\right) & \\
= & \left(\hat{G}^{L}\left(\mathbf{x}_{A}, \mathbf{x}_{1}\right) \cdots \hat{G}^{L}\left(\mathbf{x}_{A}, \mathbf{x}_{M}\right)\right) \\
& \times\left(\begin{array}{ccc}
\hat{p}^{L}\left(\mathbf{x}_{1}, \mathbf{x}_{S}^{(1)}\right) & \ldots & \hat{p}^{L}\left(\mathbf{x}_{1}, \mathbf{x}_{S}^{(N)}\right) \\
\vdots & \ddots & \vdots \\
\hat{p}^{L}\left(\mathbf{x}_{M}, \mathbf{x}_{S}^{(1)}\right) & \ldots & \hat{p}^{L}\left(\mathbf{x}_{M}, \mathbf{x}_{S}^{(N)}\right)
\end{array}\right),
\end{aligned}
$$

where we made use of the Berkhout (1982) matrix-vector notation and the frequency dependency was omitted for brevity. We can rewrite this equation as

$$
\hat{\mathbf{p}}_{A}^{L}=\hat{\mathbf{g}}^{L} \hat{\mathbf{P}}_{B}^{L}
$$

For $N$ sources on the ground surface and $M$ receivers in well $2, \hat{\mathbf{P}}_{B}^{L}$ forms an $M \times N$ matrix whose columns contain $\hat{p}^{L}\left(\mathbf{x}, \mathbf{x}_{S}, \omega\right)$ for a fixed source position $\left(\mathbf{x}_{S}\right)$ and variable receiver positions $(\mathbf{x})$ in well 2 (i.e., this becomes a shot gather), and whose rows contain $\hat{p}^{L}\left(\mathbf{x}, \mathbf{x}_{S}, \omega\right)$ for a fixed receiver position $(\mathbf{x})$ and variable source positions $\left(\mathbf{x}_{S}\right)$ (i.e., a receiver gather). Hereafter, we call the $\hat{\mathbf{P}}_{B}^{L}$ matrix the incident field matrix. On the other hand, $\hat{\mathbf{p}}_{A}^{L}$ is a $1 \times N$ row vector containing $\hat{p}^{L}\left(\mathbf{x}_{A}, \mathbf{x}_{S}, \omega\right)$ for a fixed receiver position $\left(\mathbf{x}_{A}\right)$ and variable source positions $\left(\mathbf{x}_{S}\right)$. Similarly, $\hat{\mathbf{g}}^{L}$ is a $1 \times M$ row vector containing $\hat{G}^{L}\left(\mathbf{x}_{A}, \mathbf{x}, \omega\right)$ for a fixed receiver position $\left(\mathbf{x}_{A}\right)$ and variable borehole source positions $(\mathbf{x})$, which is the Green's function that we wish to retrieve.

To estimate the unknown wavefield $\hat{\mathbf{g}}^{L}$ from equation 9, we use matrix inversion so that

$$
\hat{\mathbf{g}}^{L}=\hat{\mathbf{p}}_{A}^{L}\left(\hat{\mathbf{P}}_{B}^{L}\right)^{-1},
$$

where $\left(\hat{\mathbf{P}}_{B}^{L}\right)^{-1}$ is the generalized inverse of $\hat{\mathbf{P}}_{B}^{L}$ (Menke, 1989). Equation 10 shows that if surface sources have different wavelets at different positions (e.g., $\left.\hat{p}^{L}\left(\mathbf{x}_{A}, \mathbf{x}_{S}^{(k)}, \omega\right)=\hat{S}\left(\mathbf{x}_{S}^{(k)}, \omega\right) \hat{G}^{L}\left(\mathbf{x}_{A}, \mathbf{x}_{S}^{(k)}, \omega\right)\right)$, MDD implicitly accounts for them and retrieves the Green's function. Equation 7 implies that stable estimation of $\hat{G}^{L}\left(\mathbf{x}_{A}, \mathbf{x}, \omega\right)$ requires observation of $\hat{p}^{L}\left(\mathbf{x}_{A}, \mathbf{x}_{S}, \omega\right)$ and $\hat{p}^{L}\left(\mathbf{x}, \mathbf{x}_{S}, \omega\right)$ from sources that are sufficiently widely spread. However, for practical application, the number of sources is finite and their distribution is limited, so equation 10 could become an ill-posed problem and make it difficult to estimate $\hat{\mathbf{g}}^{L}$ uniquely. Therefore, Wapenaar et al. (2008b) stabilize the gener- 
alized inverse $\left(\hat{\mathbf{P}}_{B}^{L}\right)^{-1}$ by using damped-least-squares inversion as follows:

$$
\left(\hat{\mathbf{P}}_{B}^{L}\right)^{-1}=\left(\hat{\mathbf{P}}_{B}^{L}\right)^{\dagger}\left[\hat{\mathbf{P}}_{B}^{L}\left(\hat{\mathbf{P}}_{B}^{L}\right)^{\dagger}+\epsilon^{2} \mathbf{I}\right]^{-1}
$$

where $\epsilon$ is Tikhonov's regularization parameter (Morozov et al., 1984), which stabilizes the inverse matrix on the right-hand side of equation 11 , and $\mathbf{I}$ is a unit matrix. The dagger symbol $\dagger$ indicates Hermitian conjugation. Because equation 11 is evaluated for all frequencies, the appropriate value of $\epsilon$ is frequency dependent. When $\epsilon$ is too small, the solution of equation 11 is unstable and gives rise to artifacts. When $\epsilon$ is too large, the solution of equation 11 is overdamped and its resolution is low. Therefore, to obtain a high-quality Green's wavefield, it is essential to estimate a suitable value of $\epsilon$ at every frequency. Because $\epsilon$ may depend on both source-receiver configurations and data acquisition noise, it is difficult to specify an optimal value. We propose a different approach, as discussed below.

To obtain a stable solution for equation 9 , we adopt a singular-value decomposition (SVD) scheme. SVD is a powerful tool for the solution of ill-posed linear inverse problems (Klema and Laub, 1980). Because the least-squares method using SVD requires the division of singular values, it is easy to evaluate how the retrieved wavefield $\hat{\mathbf{g}}^{L}$ is unstably estimated. When the rank of the incident field matrix $\hat{\mathbf{P}}_{B}^{L}$ is $r=\operatorname{rank}\left(\hat{\mathbf{P}}_{B}^{L}\right) \leq \min (N, M)$, we can use SVD to decompose $\hat{\mathbf{P}}_{B}^{L}$ as follows:

$$
\hat{\mathbf{P}}_{B}^{L}=\mathbf{V}_{r} \boldsymbol{\Delta}_{r} \mathbf{U}_{r}^{\dagger}
$$

where $\boldsymbol{\Delta}_{r}$ is an $r \times r$ diagonal matrix whose diagonal components are nonzero singular values. The symbol $\mathbf{V}_{r}$ is an $M \times r$ matrix, the columns of which are composed of the $r$-eigenvectors of $\hat{\mathbf{P}}_{B}^{L}\left(\hat{\mathbf{P}}_{B}^{L}\right)^{\dagger}$ having nonzero eigenvalues. The symbol $\mathbf{U}_{r}$ is an $N \times r$ matrix, the columns of which are composed of the r-eigenvectors of $\left(\hat{\mathbf{P}}_{B}^{L}\right)^{\dagger} \hat{\mathbf{P}}_{B}^{L}$ having nonzero eigenvalues. Now that we have the pseudoinverse of $\hat{\mathbf{P}}_{B}^{L}$ as

$$
\left(\hat{\mathbf{P}}_{B}^{L}\right)^{-1}=\mathbf{U}_{r} \boldsymbol{\Delta}_{r}^{-1} \mathbf{V}_{r}^{\dagger}
$$

the solution of equation 9 becomes

$$
\hat{\mathbf{g}}_{\mathrm{est}}^{L}=\hat{\mathbf{p}}_{A}^{L} \mathbf{U}_{r} \boldsymbol{\Delta}_{r}^{-1} \mathbf{V}_{r}^{\dagger}
$$

By evaluating equation 14 for all frequencies, we retrieve new spectra of the crosswell Green's function $\hat{G}^{L}\left(\mathbf{x}_{A}, \mathbf{x}, \omega\right)$ as a common-receiver gather.

An incident field matrix is constructed for each frequency, and the maximum singular values differ for each frequency. The magnitude of the singular value corresponds to the energy of the system and the maximum singular value at each frequency is assumed to be proportional to the magnitude of the energy of the observed data. As previously mentioned, MDD commonly becomes an ill-posed problem when applied to field data, but this also is the case for synthetic data, so the incident field matrix $\hat{\mathbf{P}}_{B}^{L}$ will not be a full-rank matrix. Hence, several singular values that are zero or very small compared to the maximum value typically are excluded (e.g., Klema and Laub, 1980; Ursin and Zheng, 1985; Freire and Ulrych, 1988). For MDD, it is better to exclude singular values that are small compared to the maximum value for all frequencies.

\section{APPLICATION TO MODELED DATA}

\section{Model description}

We apply the CC method (equation 5) and the MDD method (equation 14) to modeled data and compare the results. The velocity model we use to model the data is based on the logged P-wave velocity of the field data discussed in the following section.

Two vertical boreholes are used, each with 72 receivers installed at intervals of $2 \mathrm{~m}$ between $28 \mathrm{~m}$ and $170 \mathrm{~m}$ depth. These receiver arrays are used to record wavefields from surface energy sources. With interferometry, we retrieve crosswell wavefields whose virtual sources are chosen to be in well 2 . The distribution of surface sources is important for both the CC and MDD methods. We use the same surface-source distribution for the CC and MDD methods to facilitate comparison of the two.

As we aim at retrieval of responses at well 1 from virtual sources in well 2 using the MDD method, we place our surface sources only to the right of well 2 (Figure 1). Because the velocity model is $1 \mathrm{D}$ in the vertical direction, all wavefields recorded in the boreholes from surface sources, lying to the right of well 2, are left-propagating. Therefore, we do not need to apply wavefield separation and we can use the total wavefield for MDD. Note that for conventional VSP or crosswell processing, the up-down wavefield separation is performed, whereas left-right separation is not common. It would be difficult to see prominent left- or right-propagating waves unless a prominent vertical or steeply dipping reflector is present. Nevertheless, a layered subsurface without strong changes of the seismic parameters in the horizontal direction would effectively give rise to left- or right-propagating fields from a smart choice of surface sources; this would be very beneficial for the application of SI by MDD.

The appropriateness of this one-sided source distribution for the CC method can be explained as follows: Stationary-phase theory for the CC method (e.g., Schuster et al., 2004; Snieder, 2004; Snieder et al., 2006) allows us to consider a hypothetical reflection raypath that propagates from the receiver position in well 2 to the receiver position in well 1. If this hypothetical raypath originates at a surface source, then it can be said that this source lies at the stationary-phase point (is a stationary source) with respect to the retrieval of crosswell reflections. In our velocity model (one-dimensional in the vertical direction, Figure 1), the stationary sources that produce causal crosswell reflections are all to the right of well 2. Therefore, it is reasonable to install surface sources at the right side of well 2. Surface sources lying to the left of well 1 would produce acausal reflections. Sources lying between the boreholes would contribute to the destructive interference of crosscorrelations (Snieder et al., 2006; Mehta et al., 2008b; Schuster, 2009).

We model 51 surface sources placed at 2-m intervals in a line starting $1 \mathrm{~m}$ from well 2 and extending $101 \mathrm{~m}$ to the right. Wavefields are modeled using an acoustic finite-difference time domain (FDTD) method (Virieux, 1986). The modeled record length is $0.4 \mathrm{~s}$ and the sample interval is $0.2 \mathrm{~ms}$. The source wavelet is a Ricker wavelet with a central frequency of $80 \mathrm{~Hz}$. Gaussian noise is added to the modeled data. The modeled shot gather recorded at well 1 from the surface source $1 \mathrm{~m}$ from well 2 (Figure 2) shows that there are both direct waves and reflections that originate from subsurface layers.

\section{Characteristics of the rank of the incident field matrix}

We construct the matrix and vectors in equation 9 for the numerically modeled data by Fourier transformation and apply SVD to the 
incident field matrix $\hat{\mathbf{P}}_{B}^{L}$ for all frequencies (Figure 3a). For each frequency, $r=\operatorname{rank}\left(\hat{\mathbf{P}}_{B}^{L}\right) \leq \min (N, M)$, so Figure 3 a shows 51 singular values, which is the number of sources $(N=51)$. The magnitudes of the singular values shown in Figure $3 \mathrm{a}$ are the diagonal components of $\boldsymbol{\Delta}_{r}$ ordered in descending magnitude. The frequency interval for this calculation is $2.5 \mathrm{~Hz}$ and the Nyquist frequency is $2500 \mathrm{~Hz}$. However, the singular values are evaluated only as high as a frequency of $300 \mathrm{~Hz}$ because we consider there would be very little signal above $300 \mathrm{~Hz}$ as can be expected for an $80 \mathrm{~Hz}$ central-frequency Ricker wavelet. Therefore, the data at frequencies above $300 \mathrm{~Hz}$ are not used for the MDD calculation.

The singular values at $100 \mathrm{~Hz}$ (Figure 3b) show that they decay approximately linearly, but with two different slopes. At the beginning, from first singular value to seventh singular value, the slope is steep and these singular values rapidly diminish in amplitude. After about the 44 singular values, the slope is less steep, but the amplitudes already are too low in comparison to the maximum amplitude. This indicates that our problem is ill-posed and the incident field matrix is not a full-rank matrix.

If the wavefield sources were both at the surface and in the subsurface, the available rank of $\hat{\mathbf{P}}_{B}^{L}$ would increase. To confirm this, we model wavefield data from subsurface sources and apply SVD to these data. For this modeling, we use the same receiver configuration as for the surface-source simulation (Figure 1) and place 51 sources at 2-m depth intervals from 0 to $100 \mathrm{~m}$ in a vertical well $50 \mathrm{~m}$ to the right of well 2. The presence of the free surface would effectively mean a doubling of the source aperture in the direction above the sources as a result of the interpretation of the free-surface reflections as wavefields from mirrored sources. Because the recording times, though, are kept the same, $0.4 \mathrm{~s}$, the aperture would not increase so dramatically. We calculate wavefields for this source-receiver configuration and add the same Gaussian noise as that used for the surface-source simulation. Hereafter, we refer to this data set as the subsurface-source record. We calculate the singular values of the incident field matrix for this subsurface-source record (Figure 4a).

Even though the sources are in the subsurface, the incident field

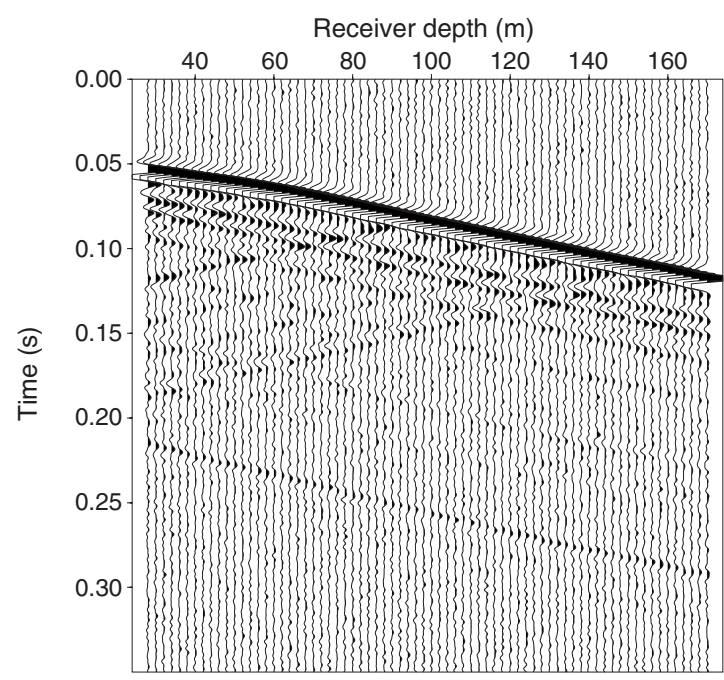

Figure 2. Example of data modeled using a finite-difference timedomain method. The data for this shot gather are recorded in well 1 from the first surface source to the right of well 2. Gaussian noise is added to the modeled data after normalization to rms amplitude. The signal-to-noise ratio is set to 40 . matrix still is not full rank, possibly because of the sparse distribution of sources. However, the subsurface-source record (Figure 4a) has a higher number of singular values with an amplitude of $5 \%$ or more of the global maximum singular value than is the case for the surface-source record (Figure 3a).

For the subsurface-source record, the singular values at $100 \mathrm{~Hz}$ (Figure 4b) do not reduce linearly, as they do for the surface-source record (Figure 3b), and the first 11 singular values are larger than those of the surface-source record (Figure $3 b$ ). Therefore, the contributions of these singular values to the pseudoinverse calculation are larger than for the surface-source record because the strength of the correlation of the incident field matrix is decreased by the source distribution.

That $\hat{\mathbf{P}}_{B}^{L}$ is not a full-rank matrix can be confirmed from the crosscorrelation matrices $\hat{\mathbf{P}}_{B}^{L}\left(\hat{\mathbf{P}}_{B}^{L}\right)^{\dagger}$ and $\left(\hat{\mathbf{P}}_{B}^{L}\right)^{\dagger} \hat{\mathbf{P}}_{B}^{L}$. High values of these crosscorrelations in the off-diagonal elements indicate linear dependence of the rows or columns of the $\hat{\mathbf{P}}_{B}^{L}$ matrix, which reduces the rank of $\hat{\mathbf{P}}_{B}^{L}$. Because the $\hat{\mathbf{P}}_{B}^{L}$ matrix is formed from observed data, the two crosscorrelation matrices $\hat{\mathbf{P}}_{B}^{L}\left(\hat{\mathbf{P}}_{B}^{L}\right)^{\dagger}$ and $\left(\hat{\mathbf{P}}_{B}^{L}\right) \hat{\mathbf{P}}_{B}^{L}$ provide measures of the correlation between receiver gathers and between shot gathers, respectively. Therefore, the strength of the correlation of $\hat{\mathbf{P}}_{B}^{L}$ (and the rank of $\hat{\mathbf{P}}_{B}^{L}$ ) may depend on the source or receiver distributions. In other words, the lower rank of the matrix means that the available sources do not illuminate the receiver array sufficiently.

To determine the strength of the correlation of the column compo-

a)

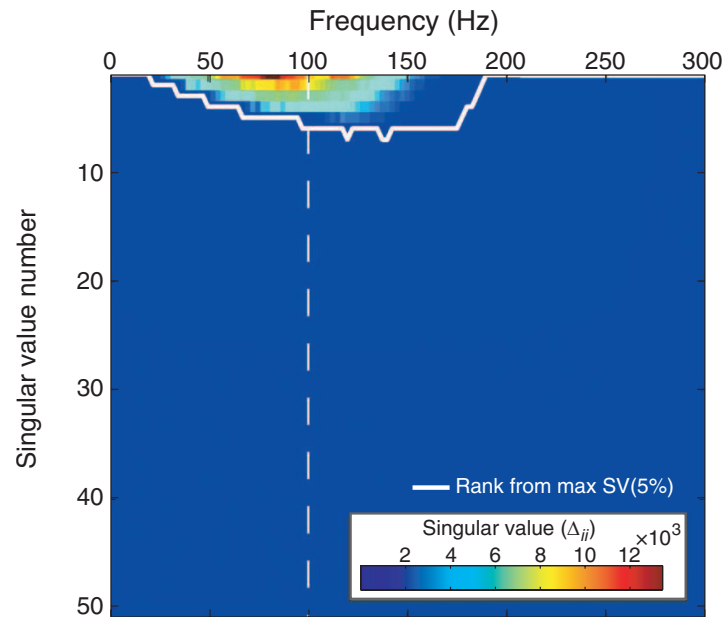

b)

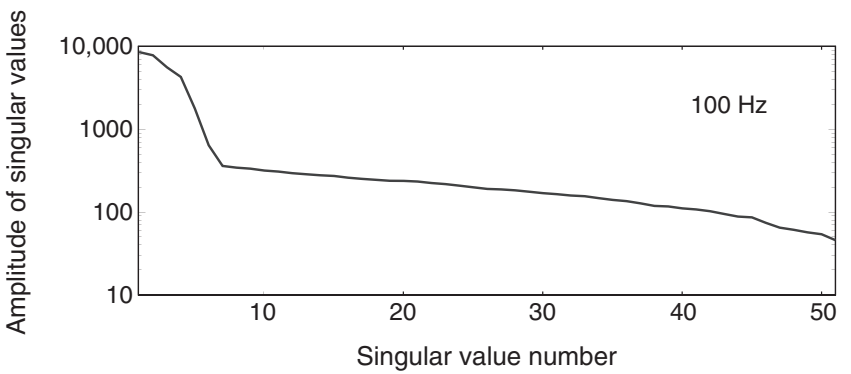

Figure 3. Singular values $\Delta_{i i}$ of the incident field matrix $\hat{\mathbf{P}}_{B}^{L}$ from surface-source data. (a) Singular values of $\hat{\mathbf{P}}_{B}^{L}$ at each frequency. The solid white line shows the rank determined from the $5 \%$ criteria of the global maximum. (b) Singular values of $\hat{\mathbf{P}}_{B}^{L}$ at $100 \mathrm{~Hz}$ as indicated by the dashed line in (a). 
nents of the incident field matrix (crosscorrelation between shot gathers in well 2 from different sources) we calculate the crosscorrelation matrix $\left(\hat{\mathbf{P}}_{B}^{L}\right)^{\dagger} \hat{\mathbf{P}}_{B}^{L}$ and scale it to represent the crosscorrelation coefficient as

$$
\mathbf{R}(i, j)=\frac{\mathbf{V}(i, j)}{\sqrt{\mathbf{V}(i, i) \mathbf{V}(j, j)}},
$$

where $\mathbf{V}$ is a variance-covariance matrix of shot gathers (variance and covariance of the column components of $\hat{\mathbf{P}}_{B}^{L}$ ) and $\mathbf{R}$ represents the coherence matrix, which is a crosscorrelation coefficient matrix scaled by the standard deviations of each shot gather. The $i$ th-row and $j$ th-column components of Figure 5 a indicate the absolute values of the coherence $(\mathbf{R}(i, j))$ for shot gathers in well 2 whose (surface or subsurface) sources are at $\mathbf{x}_{S}^{(i)}$ and $\mathbf{x}_{S}^{(j)}$. Therefore, the diagonal components of the crosscorrelations (Figure 5a) all are equal to one. If there are many components with large coherence in the off-diagonal elements, the observed data resemble each other and the available rank of the incident field matrix is low.

The coherence of the incident field matrix for the surface-source record (Figure 5a) shows a strong correlation along the vicinity of the diagonal components (warm-colored area). This indicates that the shot gathers from adjacent surface sources correlate well, possibly because of the simple velocity model we used.

We show the coherence matrix of the incident field matrix for the subsurface-source record (Figure 5b). Here, the gridded structure

a)

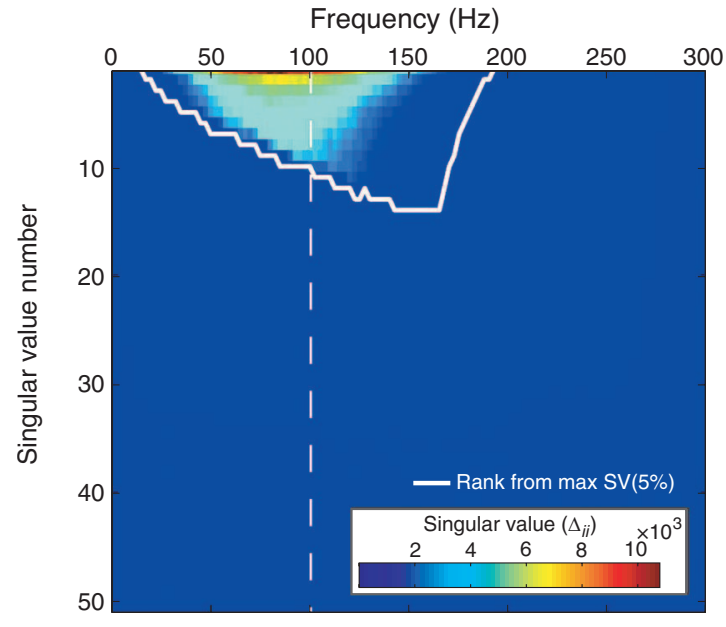

b)

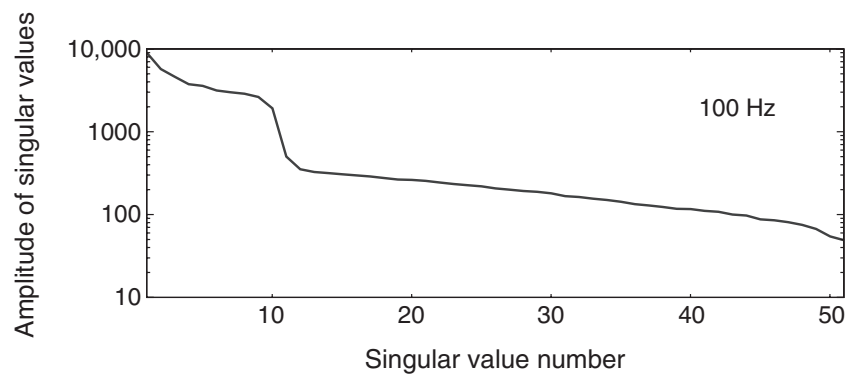

Figure 4. Singular values $\Delta_{i i}$ of the incident field matrix $\hat{\mathbf{P}}_{B}^{L}$ from subsurface-source data. (a) Singular values of $\hat{\mathbf{P}}_{B}^{L}$ at each frequency. The white solid line shows the rank determined from the $5 \%$ criteria of the global maximum. (b) Singular values of $\hat{\mathbf{P}}_{B}^{L}$ at $100 \mathrm{~Hz}$ as indicated by the dashed line in (a). appearing in Figure $5 \mathrm{~b}$ comes from the interference of events reflected with positive and negative reflection coefficients. The strongest contributor to this structure is the free surface, whose reflection coefficient is -1 . In Figure 5a, this gridding is not visible because the sources are located at the free surface in this situation. The coherence matrix of the incident field matrix for the subsurface-source record (Figure 5b) shows a narrower diagonal correlated area than that of the surface-source record (Figure 5a). This indicates that the shot gathers from vertically adjacent sources are less well correlated. There are 401 components of the surface-source record with coherence that exceeds 0.9 , whereas only 119 components of the subsurface-source record are in that category (Figure 5a and b, respectively).

The above observations confirm that subsurface sources provide a higher-ranked incident field matrix than surface sources, and thus provide a larger amount of data that contribute to retrieved crosswell wavefields. The simple horizontally layered velocity structure we use in this study contributes to the strong correlation of the surfacesource record. The strength of the correlation of observed data would be dependent on the velocity model. Therefore, for a more complex velocity model, the rank of the incident field matrix would increase because the strength of the correlations between data is reduced by the complex raypaths that result from surface sources. The multiple scattering from the subsurface complexities would increase the receivers' illumination from different directions and effectively would add extra secondary sources in the subsurface. However, for a more

a)

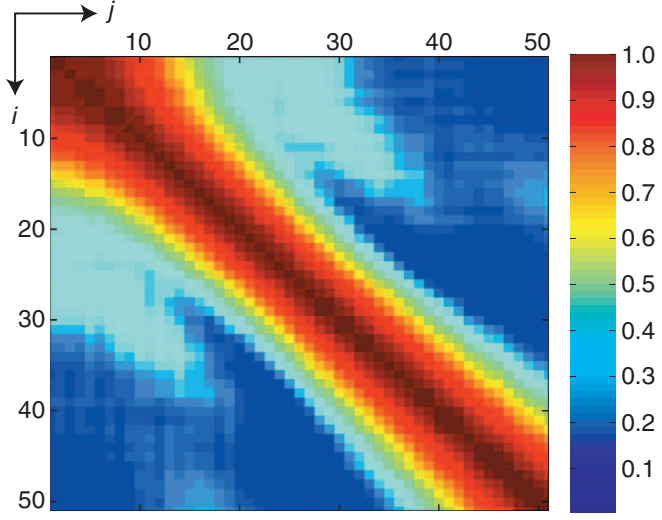

\begin{tabular}{l}
0 \\
0 \\
$\frac{0}{0}$ \\
$\frac{10}{0}$ \\
$\frac{1}{0}$ \\
0 \\
40 \\
0 \\
$\frac{0}{\pi}$ \\
$\frac{1}{0}$ \\
0 \\
5 \\
\hline 0 \\
0 \\
$\frac{10}{4}$
\end{tabular}

b)

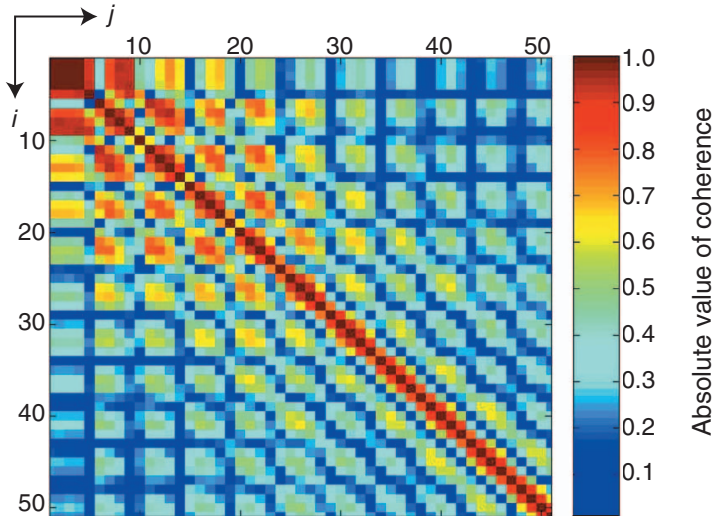

Figure 5. Absolute value of the coherence matrix of the incident field matrix. Scaled $\left(\hat{\mathbf{P}}_{B}^{L}\right) \hat{\mathbf{P}}_{B}^{L}$ for $100 \mathrm{~Hz}$ from (a) surface-source data and (b) subsurface-source data. The indices $i$ and $j$ indicate the source positions $\mathbf{x}_{S}^{(i)}$ and $\mathbf{x}_{S}^{(j)}$. 
complex velocity model, separation of the left-going wavefield, which is a condition for MDD, might be required. Furthermore, complexity might introduce common stationary noise, e.g., scattering from a point-like scatterer (in relation to the dominant wavelength) would introduce energy that illuminates the receivers from the same direction irrespective of the position of the surface sources. Such cases, though, would not be frequent.

\section{Imaging results from crosscorrelation and multidimensional deconvolution}

We retrieve crosswell reflection wavefields from surface-source records using the $\mathrm{CC}$ and MDD methods. We use as surface-source records the acoustically modeled data inside two boreholes, each with 72 receivers installed at intervals of $2 \mathrm{~m}$ between $28 \mathrm{~m}$ and $170 \mathrm{~m}$ depth. We model 51 surface sources placed at 2-m intervals in a line starting $1 \mathrm{~m}$ from well 2 and extending $101 \mathrm{~m}$ to the right. For our retrieval using the MDD algorithm, the rank of the incident field matrix $\hat{\mathbf{P}}_{B}^{L}$ is determined taking into account only those values that are larger than $5 \%$ of the maximum value (Figure $3 \mathrm{a}$ ). We estimate the crosswell Green's function $\hat{G}^{L}\left(\mathbf{x}_{A}, \mathbf{x}, \omega\right)$ as a receiver gather from equation 14.

Figure 6a shows the crosswell common-receiver gather retrieved by MDD in the time domain for the receiver at $106 \mathrm{~m}$ depth in well 1. For comparison, we retrieve the crosswell common-receiver gather by using the $\mathrm{CC}$ method (Figure $6 \mathrm{~b}$ ) with equation 5 . The receiver gather retrieved by the $\mathrm{CC}$ method (Figure $6 \mathrm{~b}$ ) is wavelet-deconvolved after crosscorrelation and summation (equation 5). We directly model a wavefield for a receiver placed at $106 \mathrm{~m}$ depth in well 1 and sources placed at 2-m depth intervals from $28 \mathrm{~m}$ to $170 \mathrm{~m}$ in well 2 (Figure 6c). The responses in Figure 6c also are waveletdeconvolved. Each of the three receiver gathers shows a free-surface reflection ( $\mathrm{r} 1$, downgoing reflection), a reflection from the boundary at $160 \mathrm{~m}$ depth ( $\mathrm{r} 2$, upgoing reflection), and a reflection from the boundary at $40 \mathrm{~m}$ depth ( $\mathrm{r}$, downgoing reflection). Here, by upgoing (or downgoing) reflections, we mean reflected waves that propagate upward (or downward) from the acoustic impedance boundary that causes their reflection. The direct arrivals below about $100 \mathrm{~m}$ were not retrieved in either the MDD (Figure 6a) or CC (Figure 6b) results. This is because there were no upgoing waves contributing to the retrieval of those direct arrivals.

The $\mathrm{CC}$ result is dominated by upgoing waves, whereas the MDD result contains both up and down events with better relative amplitudes. For example, the downgoing waves ( $\mathrm{r} 1$ and $\mathrm{r} 3$ ) are appearing in the MDD results with better amplitudes than in the $\mathrm{CC}$ results. Because the $\mathrm{CC}$ result is dominated by upgoing waves, $\mathrm{r} 3$ is covered by downgoing events and cannot be recognized in Figure 6b. The upgoing events ( $\mathrm{r} 2$ ) are retrieved well in both the MDD and $\mathrm{CC}$ results. The MDD result (Figure 6a) and CC result (Figure 6b) exhibit comparable resolution because MDD directly retrieves Green's functions, whereas the $\mathrm{CC}$ result is deconvolved by a wavelet that is the autocorrelation of the original wavelet of the surface sources.

The fact that we use sources only at the surface means that we create a preferred direction of illumination at the receiver arrays from above, that is, for upgoing reflections. For the retrieval of downgoing reflections, we rely on contributions from waves that have reflected at subsurface layers, and thus act as secondary Huygens sources, before being recorded by the receivers in well 2. Such waves are at least second-order reflections of relatively low energy. Furthermore, equation 1 is obtained after a far-field approximation.
For secondary sources close to the receivers, this approximation is not valid anymore and might result in significant amplitude errors.

The above reasons could explain why the $\mathrm{CC}$ result in Figure $6 \mathrm{~b}$ exhibits mainly upgoing retrieved events. On the other hand, the MDD method would treat the amplitudes of both upgoing (r2) and downgoing reflections ( $\mathrm{r} 1$ and $\mathrm{r} 3$ ) more accurately and would give better results (Figure 6a). This happens because the MDD method is more robust with respect to the source distribution than the CC method as shown in Wapenaar et al. (2008b).

We image the subsurface structure using retrieved crosswell wavefields. We retrieve all the crosswell wavefields (i.e., all crosswell receiver gathers). Because there are 72 receivers in well 1, we apply MDD (equation 14) 72 times. The total crosswell wavefields retrieved from MDD contain $72 \times 72$ traces. For imaging, retrieved crosswell data are decomposed into upgoing and downgoing reflections. Upgoing reflections are used for imaging of the deep structures and downgoing reflections are used for imaging of the shallow structures (Lazaratos et al., 1993). We decompose the crosswell wavefield using an $f$ - $k$ filter and apply Kirchhoff prestack depth migration (Figure 7).

In the direct modeling result (Figure 7c), the images from $0 \mathrm{~m}$ to $90 \mathrm{~m}$ are produced from downgoing reflections, while the images below $90 \mathrm{~m}$ are produced from upgoing reflections. In the SI results (Figure 7a and b), we change this cut-off depth to $55 \mathrm{~m}$ because it produced better signal-to-noise ratio $(\mathrm{S} / \mathrm{N})$.

This possibly comes from the fact that in the SI, the effective propagation direction is dominated by upgoing waves. The amplitudes of these images are normalized with the amplitude at $40 \mathrm{~m}$ depth for the shallow structure (from downgoing reflections) and with the amplitude at $160 \mathrm{~m}$ depth for the deep structures (from upgoing reflections). The MDD and CC results (Figure 7a and b) agree well with the direct modeling result (Figure 7c) and the velocity model (Figure $7 \mathrm{~d}$ ). However, the images from $0 \mathrm{~m}$ to $55 \mathrm{~m}$ (from downgoing reflections) are relatively noisy. We show the migrated signals halfway between the two boreholes in Figure 8. The MDD and CC results (Figure $8 \mathrm{a}$ and $\mathrm{b}$ ) are noisier than the direct modeling result (Figure $8 \mathrm{c})$. However, the peak amplitudes below $55 \mathrm{~m}$ compare well with the direct modeling result (Figure 8c) and the reflection coefficients (Figure 8d). The amplitudes from $0 \mathrm{~m}$ to $55 \mathrm{~m}$ show the correct amplitude peaks at about $20 \mathrm{~m}$ and $40 \mathrm{~m}$ depth; however, the phases between $20 \mathrm{~m}$ and $40 \mathrm{~m}$ are not correctly imaged. This is possibly caused by the insufficient retrieval of downgoing reflections and the smearing effects of migration.

Comparing the two SI results with the direct modeling result, we can see that for the shallow reflectors, the MDD result in Figure 8a is resolved better than the $\mathrm{CC}$ result in Figure 8b, and resembles more the direct modeling result in Figure 8c. This could be because the MDD method treats the amplitude of a downgoing wave better than the CC method. For the deeper layers, both the MDD and CC methods resolved subsurface layers very well.

\section{APPLICATION TO FIELD DATA}

\section{Field data acquisition}

We compare the results of seismic interferometry applied to field data by the CC (equation 5) and the MDD (equation 14) methods. The field data are recorded in Aomori Prefecture, northeast Japan. The upper $200 \mathrm{~m}$ of the survey area are composed mainly of hori- 
zontal alternating layers of sandstone and tuff. The distance between the two boreholes and the receiver configuration is identical to that in the numerical modeling (Figure 1).

We use two boreholes, each with 72 borehole hydrophones installed between $28 \mathrm{~m}$ and 170-m-depth with an interval of $2 \mathrm{~m}$. The horizontal distance between the boreholes is $50 \mathrm{~m}$. We install explosive surface sources to the right of well 2 . Because the area available for this experiment is limited, only 13 sources are placed (at 5-m intervals) on the right side of well 2. A 24-channel hydrophone (2-m interval) cable is used three times in the borehole to provide cover-

a)

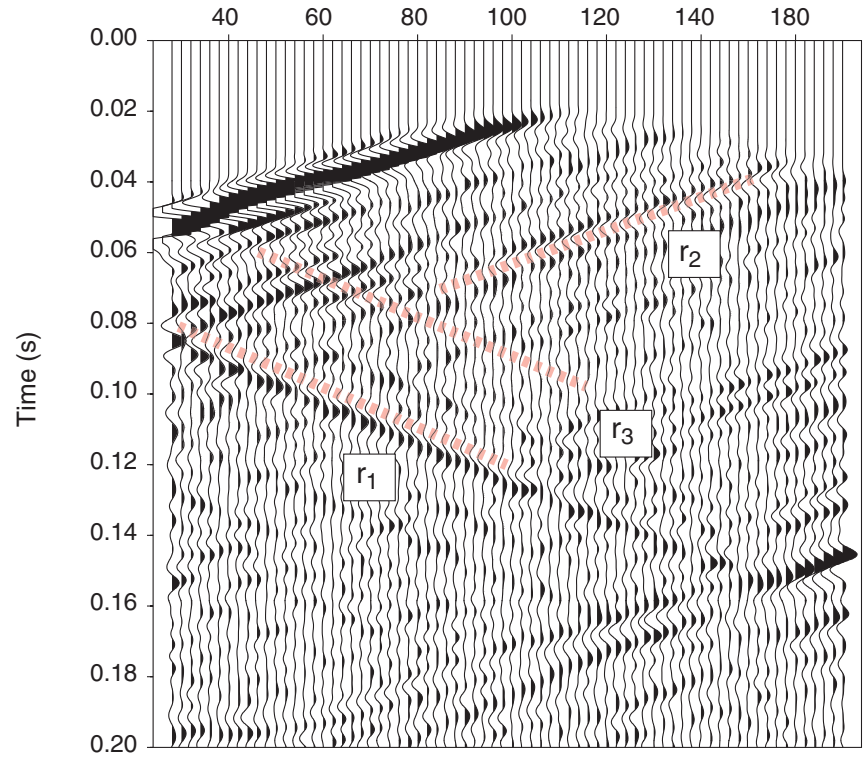

age from $28 \mathrm{~m}$ to $170 \mathrm{~m}$ depth. The temporal recording length is $0.4 \mathrm{~s}$ at a sampling rate of $0.25 \mathrm{~ms}$, as is the case for the modeled data.

To verify the crosswell wavefields retrieved from surface-source records, we acquire crosswell wavefields using a downhole nonexplosive OYO Wappa source (OWS; OYO Corporation, Tsukuba, Japan), (Ogura et al., 1992; Takahashi et al., 2001) and three-component geophones. OWS is a borehole source that generates compressional waves in a fluid with a bandwidth as high as several thousand hertz. We install 72 subsurface sources from $28 \mathrm{~m}$ to $170 \mathrm{~m}$ depth at

b)

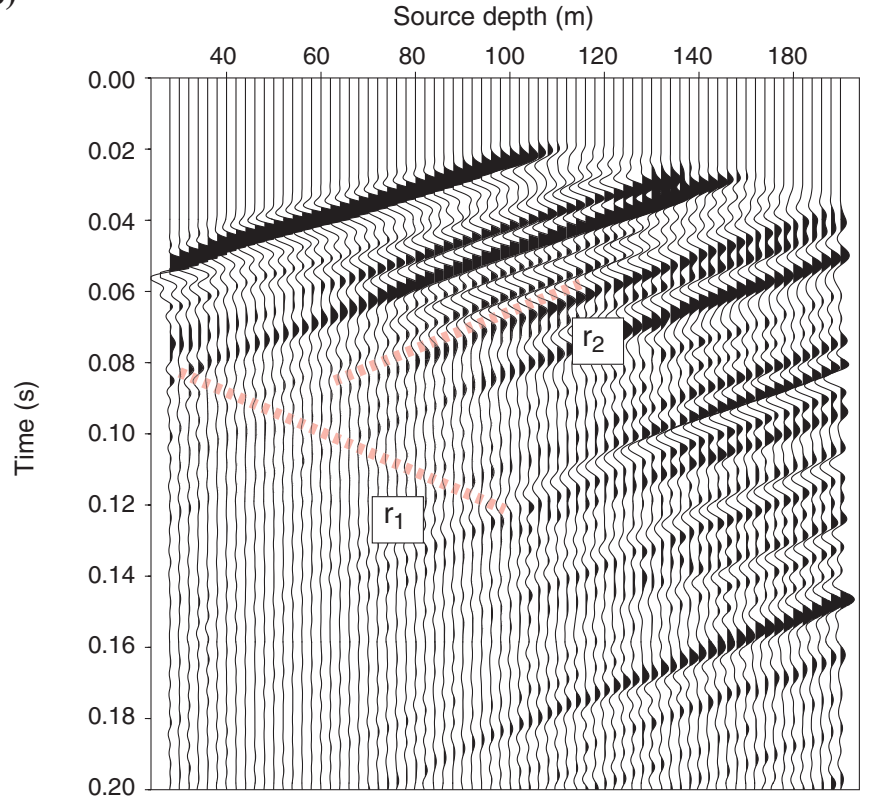

c) Source depth $(m)$

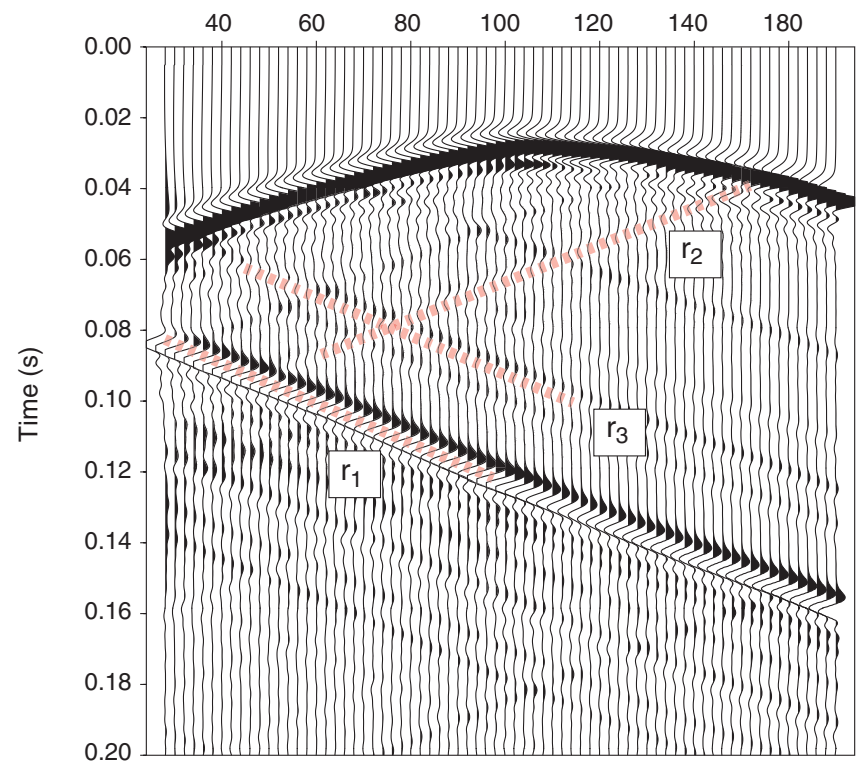

Figure 6. Synthetic crosswell receiver gather retrieved from surface sources and constructed in the time domain for a receiver at $106 \mathrm{~m}$ depth in well 1 from (a) multidimensional deconvolution, (b) wavelet-deconvolved crosscorrelation, (c) wavelet-deconvolved direct modeling of the same receiver gather with downhole sources at 2-m-depth intervals from $28 \mathrm{~m}$ to $170 \mathrm{~m}$ in well 2 . The pink dotted lines indicate a free-surface reflection ( $\mathrm{r} 1$, downgoing wave), a reflection from the boundary at $160 \mathrm{~m}$ depth ( $\mathrm{r} 2$, upgoing wave), and a reflection from the boundary at $40 \mathrm{~m}$ depth (r3, downgoing wave). Events at times earlier than the direct arrival have been muted in views (a) and (b). 
2-m intervals. Note that for the field experiment (a 3D configuration), the source boundary for $\mathrm{CC}(\partial \mathbb{D}$ in equation 4$)$ and the receiver boundary for MDD $\left(\partial \mathrm{D}_{2}\right.$ in equation 7$)$ should be a $2 \mathrm{D}$ surface. However, we still approximate them as 1D lines, as was the case for the numerical modeling, assuming that there are no significant velocity changes along the crossline direction for the boreholes.

To isolate the $\mathrm{P}$-waves in the surface-source record, we remove $\mathrm{S}$ waves and tube-waves by using an $f-k$ filter. The so-obtained P-waves are normalized to rms amplitudes (Figure 9). Both reflected waves and direct waves can be seen in Figure 9. Horizontal geological layers are predominant in this area, so we regard the observed wavefields as leftward propagating and did not need to isolate them from right-propagating wavefields before MDD.

\section{RESULTS}

We evaluate singular values of the incident field matrix $\hat{\mathbf{P}}_{B}^{L}$ at all frequencies (Figure 10a). The frequency interval for this calculation is $2.5 \mathrm{~Hz}$ and the Nyquist frequency is $2000 \mathrm{~Hz}$. We consider that signals are within the range from 0 to $400 \mathrm{~Hz}$, and calculate the singular values as high as $400 \mathrm{~Hz}$. At $100 \mathrm{~Hz}$, singular values beyond the eighth-largest value are much smaller than the maximum singular value (Figure 10b). Small singular values indicate that the incident field matrix is not full rank as was the case for the numerical modeling, so these small singular values must be truncated. We use singular values which exceed $5 \%$ of the maximum singular value (Figure 10a). a)

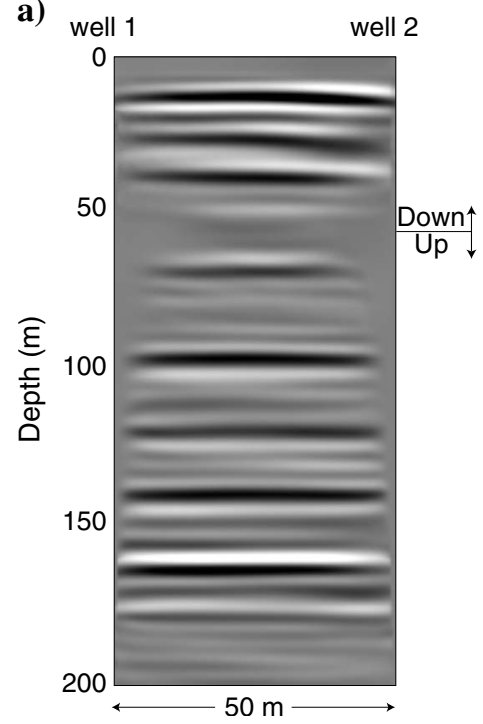

b)

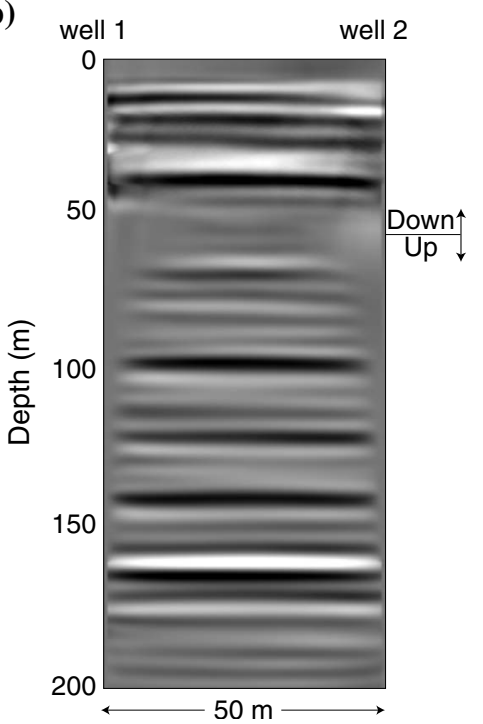

c)

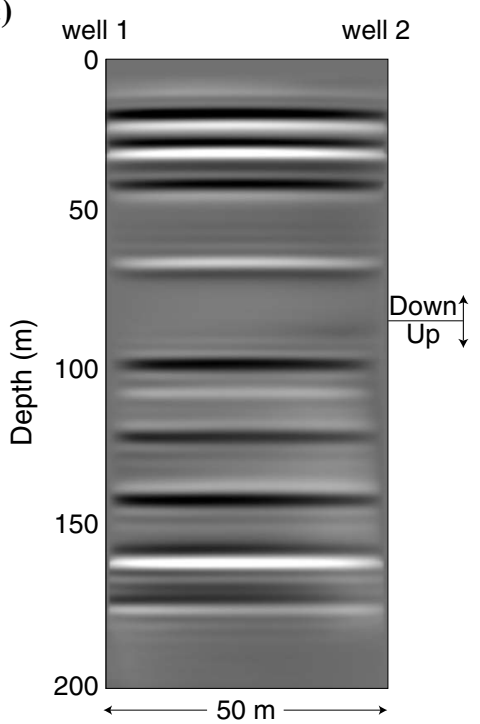

d) Velocity $(\mathrm{m} / \mathrm{s})$

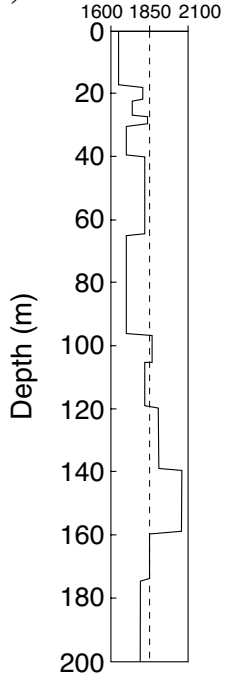

Figure 7. Results of Kirchhoff prestack migration obtained from (a) multidimensional deconvolution, (b) wavelet-deconvolved crosscorrelation, and (c) wavelet-deconvolved direct modeling. (d) The velocity model used for migration.

a) Normalized amplitude

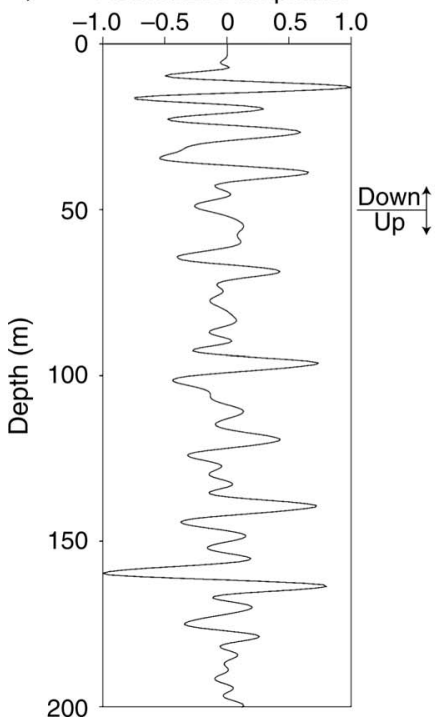

b)

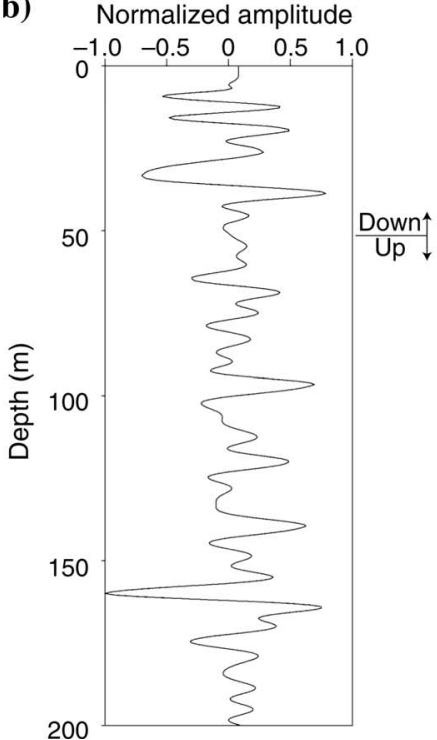

c)

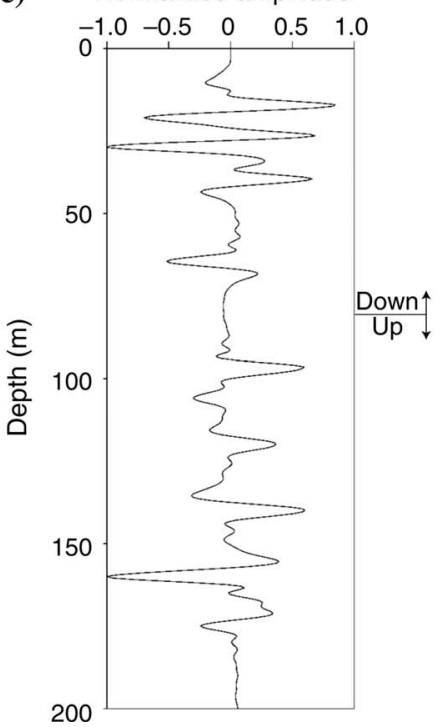

d) Normalized amplitude

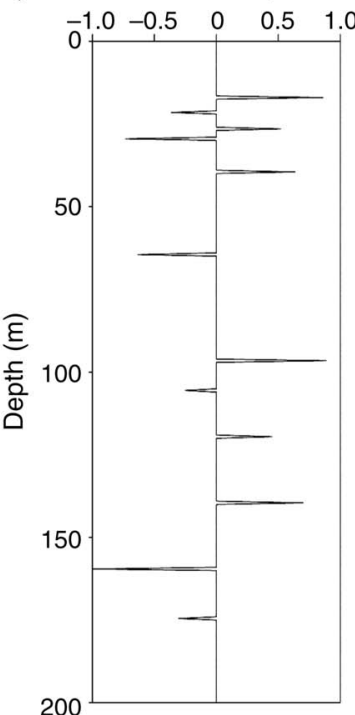

Figure 8. Migrated signals (after amplitude normalization) for the trace at the middle of the boreholes in Figure 7 from (a) multidimensional deconvolution, (b) wavelet-deconvolved crosscorrelation, and (c) wavelet-deconvolved direct modeling. (d) The refection coefficients calculated from Figure $7 \mathrm{~d}$. 
We apply the MDD method to retrieve the crosswell wavefield as a receiver gather (Figure 11a). We retrieve the equivalent receiver gather using the $\mathrm{CC}$ method (Figure 11b). The result by the $\mathrm{CC}$ method (Figure 11b) is wavelet-deconvolved after crosscorrelation and summation (equation 5). The direct arrivals below approximately $100 \mathrm{~m}$ were not retrieved in either the MDD (Figure 11a) or CC (Figure 11b) results because of the location of the sources. Comparison of these wavefields reveals that the resolution of the MDD method and the $\mathrm{CC}$ method is almost the same. However, the MDD result for the field data contains both downgoing and upgoing events, as was demonstrated with the modeled data.

In contrast, the $\mathrm{CC}$ result is dominated by upgoing events. For comparison, the receiver-gather data for the downhole source is shown (Figure 11c). Here, the horizontal displacements of the threecomponent geophone records are shown. Because the interferometric results (Figure 11a and b) are shown as an acoustic hydrophone wavefield, they differ in phase and amplitude from the wavefields recorded by the geophone. For our comparison, therefore, we focus on the traveltimes of the retrieved reflections. We can see that the traveltimes of several reflections in the wavefields retrieved by interferometry are the same as those recorded from downhole sources (Figure 11c).

We apply prestack depth migration to the total crosswell wavefields ( $72 \times 72$ traces) derived by MDD (Figure 12a), by CC (Figure $12 b$ ), and from a downhole source (Figure 12c). The images from $0 \mathrm{~m}$ to $90 \mathrm{~m}$ depth are produced from downgoing reflections and the images below $90 \mathrm{~m}$ depth are produced from upgoing reflections. The amplitudes are normalized by that of $25 \mathrm{~m}$ depth for shallow structures (from downgoing reflections) and by that of $180 \mathrm{~m}$ depth for deep structures (from upgoing reflections).

The dominant frequency of the wavefields generated by the OWS downhole source is much higher than that for the borehole records from surface sources (Figure 13). Further, the resolution of the reflection boundaries using the downhole sources (Figure 12c) is higher than the resolution obtained from interferometry. This can be explained by the very different bandwidths of the OWS and surface sources. The resolution of the wavefield retrieved from interferome-

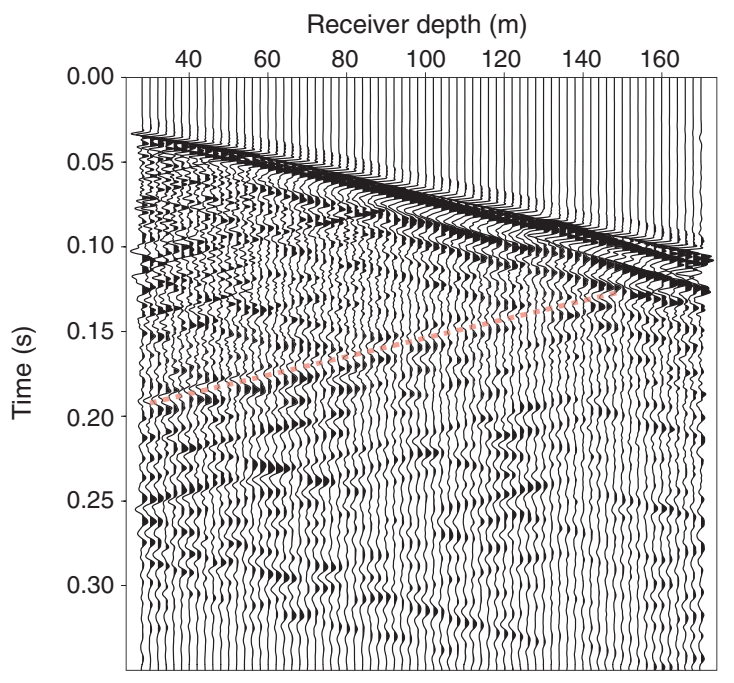

Figure 9. Example of the recorded field data after preprocessing: a shot record in well 1 from the surface source at $5 \mathrm{~m}$ distance from well 2. The pink dotted line indicates a reflection signal from a subsurface boundary. try could be improved by using large-bandwidth surface sources. Nevertheless, the reflection boundaries, for example at $25 \mathrm{~m}, 100 \mathrm{~m}$ and $180 \mathrm{~m}$ depth (arrows in Figure 12) are imaged in both the MDD and the $\mathrm{CC}$ result. Furthermore, these images agree well with the $\mathrm{P}$-wave velocity log from well 2 (Figure 12d).

\section{DISCUSSION}

We show that application of SI by MDD and by CC to crosswell data from impulsive sources at the surface can retrieve the reflection response between the two boreholes as if there were sources in one of the boreholes. Comparing the retrieved results in Figure $6 \mathrm{a}$ and $\mathrm{b}$ and Figure 11 $\mathrm{a}$ and $\mathrm{b}$ to results from an actual source in borehole well 2 (Figure 6c and Figure 11c, respectively), we can interpret several retrieved reflections. On the other hand, in Figure $6 a$ and $b$ and Figure 11a and $\mathrm{b}$ we can see different arrivals that are not present in Figure $6 \mathrm{c}$ and Figure 11c. For example, in Figure $6 \mathrm{a}$ and $\mathrm{b}$ there are arrivals close in time to $\mathrm{r} 2$ that are parallel to this reflection or which even cross it. Such arrivals are not present in Figure 6c. These are nonphysical (ghost) arrivals that appear in the retrieved results as a result of insufficient illumination by the active sources.

We show that the MDD and CC methods, applied to surfacesource records, retrieve upgoing reflection wavefields very well. Furthermore, the MDD method retrieves downgoing reflections

a)

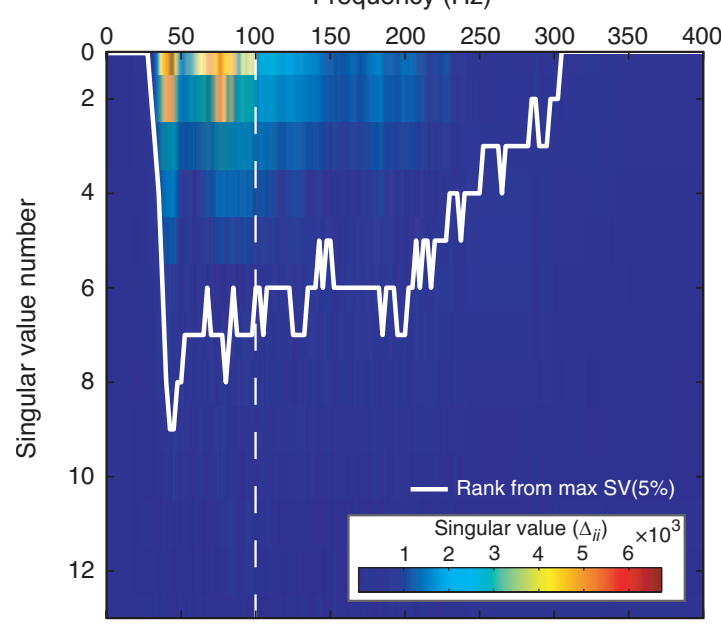

b)

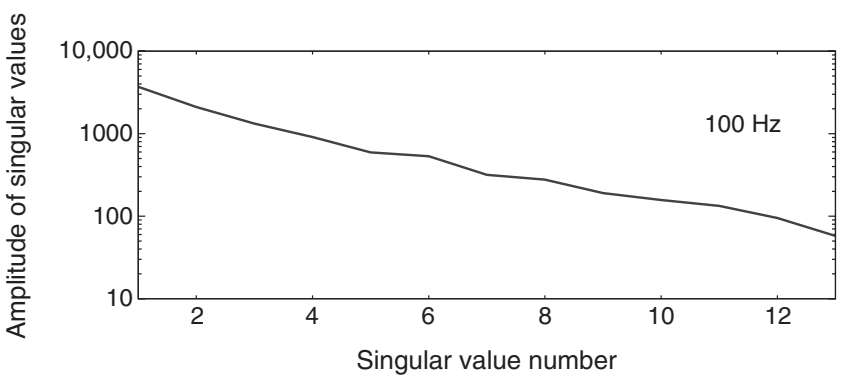

Figure 10. Singular values of the incident field matrix $\hat{\mathbf{P}}_{B}^{L}$ for the field data. Because $r=\operatorname{rank}\left(\hat{\mathbf{P}}_{B}^{L}\right) \leq \min (N, M)$, where $N$ and $M$ are the number of sources and receivers, there are 13 singular values for each frequency. (a) Singular values of $\hat{\mathbf{P}}_{B}^{L}$ at each frequency. The solid white line shows the rank determined from the 5\% criteria. (b) Singular values of $\hat{\mathbf{P}}_{B}^{L}$ at $100 \mathrm{~Hz}$ as indicated by the dashed line in (a). 

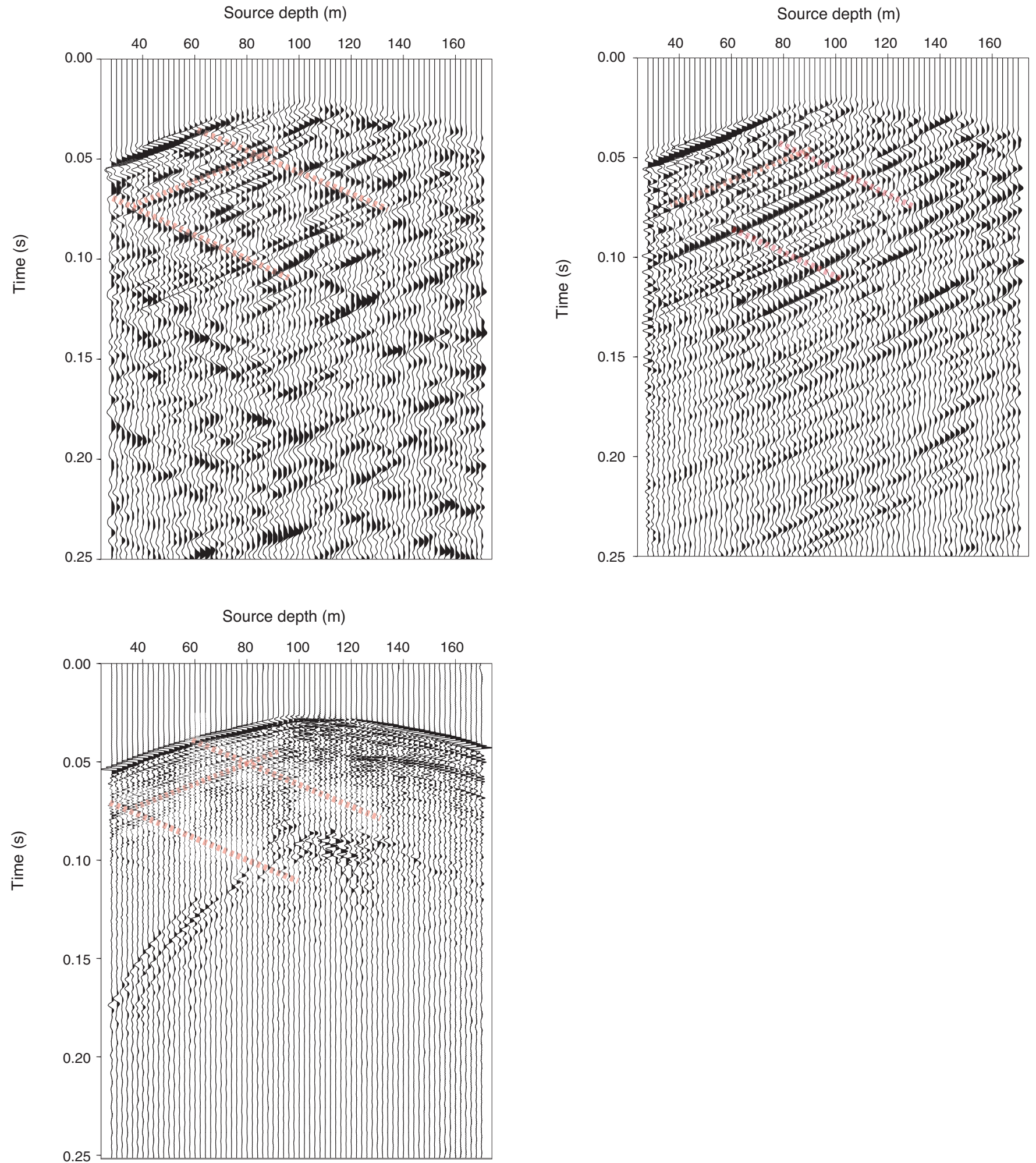

Figure 11. Crosswell receiver gather (P-waves) shown in the time domain as retrieved from surface sources for the receiver at $106 \mathrm{~m}$ depth in well 1 from (a) multidimensional deconvolution (MDD) and (b) wavelet-deconvolved crosscorrelation (CC). (c) The wavelet-deconvolved same receiver gather from a downhole source in which we observe S-waves. The pink dotted lines indicate reflection events retrieved by MDD and CC that correspond to the downhole source. Events at times earlier than the direct arrival have been muted in views (a) and (b). 
a)

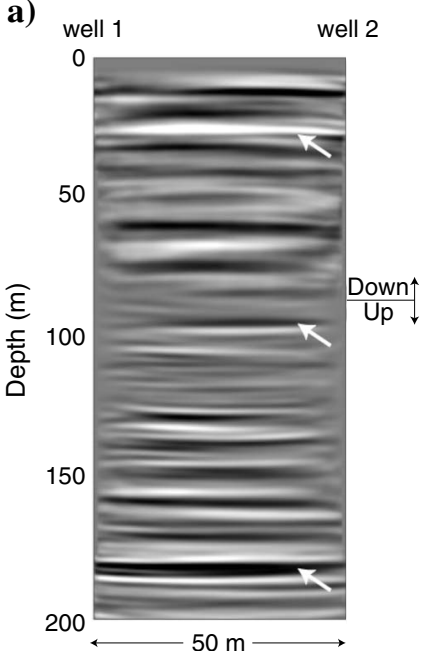

b)

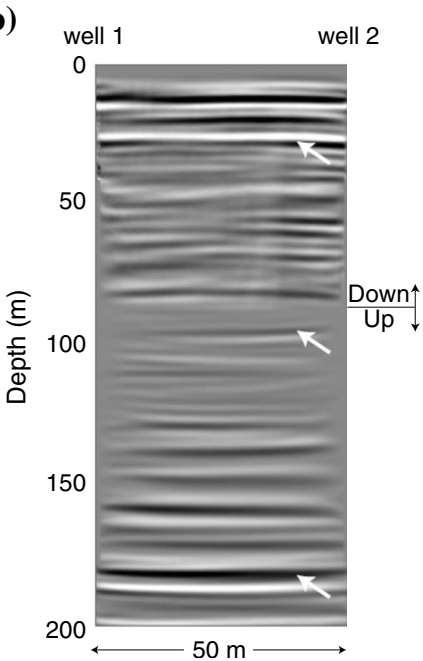

c)

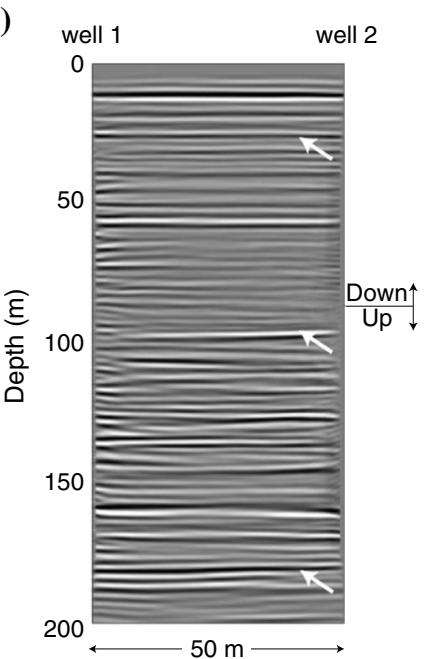

d)
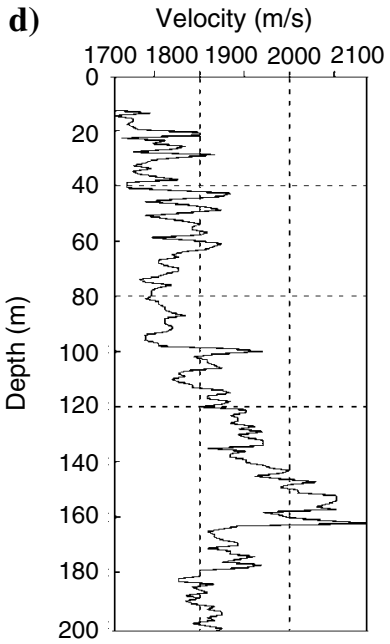

Figure 12. Results of Kirchhoff prestack migration obtained from (a) multidimensional deconvolution and (b) wavelet-deconvolved crosscorrelation. (c) Migrated output from downhole sources and (d) P-wave velocity logged at well 2. Arrows show example reflection boundaries.

with more accurate amplitudes than the CC method. However, the MDD problem for surface-source records is ill-posed, so the rank of the incident field matrix must be determined with care in this case. Because the CC method does not need a rank determination, it is simpler to achieve stable results than with the MDD method.

To compare the computational costs of the CC method and the MDD method, we measure the computation time to retrieve 72 crosswell receiver gathers using the data set of the numerical-modeling section. The computation time for the $\mathrm{CC}$ method is $30 \mathrm{~s}$, whereas that for the MDD method is $68 \mathrm{~s}$ using Intel Core i7 $(2.93 \mathrm{GHz})$ with 16 GB memory. The MDD method takes longer than the CC method because the MDD method requires SVD and a construction of the pseudoinverse matrices.

We assume a horizontally layered structure to avoid left-right decomposition for the MDD method. If the subsurface structure is more complex, the rank of the incident field matrix may be improved because the degree of correlation of the data is reduced by the complex reflection raypaths generated by surface sources. For this case, however, complexity might introduce stationary noise and, most importantly, it would be necessary to separate the wavefields before the application of MDD into left- and right-propagating wavefields.

In our field experiment, the bandwidth and resolution of the crosswell wavefield observed when using a downhole source are superior to those of the wavefields retrieved by interferometry. This is because the bandwidth of the actual wavefield is much larger than that of the surface-source record (Figure 13). Note further that the radiation pattern of the retrieved downhole virtual sources is limited by the surface-source aperture and cannot match the radiation pattern of the actual downhole sources, which emits in all directions.

The results from the $\mathrm{CC}$ method are more sensitive to the surfacesource wavelet because the dependence on the wavelet needs to be eliminated with wavelet deconvolution after the crosscorrelation. In this way, differences in the wavelets from the different surface sources and from different receiver responses might not be accounted for optimally. Contrary to this, the MDD method accounts implicitly for differences in the source wavelets during the retrieval process, and in other applications this might result in a better retrieval of the higher frequencies within the band of the energy emitted by the surface sources. On the other hand, for the MDD method, we do not

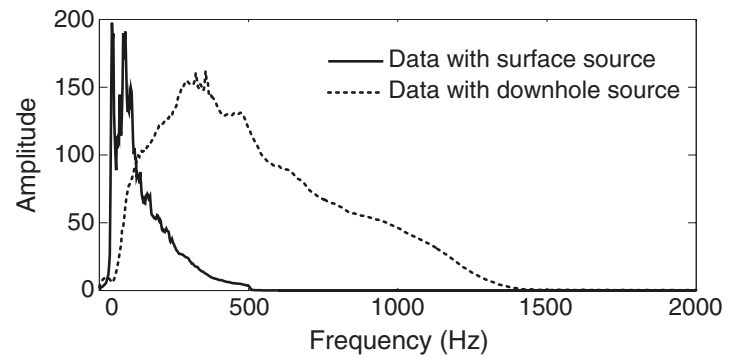

Figure 13. Average frequency spectra from input data for seismic interferometry using explosive surface sources (solid line) and crosswell data using downhole sources (dashed line).

use the high-frequency components of the seismic signal because their amplitudes are too low and get lost within the noise. Therefore, the bandwidth for our MDD results is narrow and lacks high-frequency components.

One of the reasons that the wavefields recorded with the OWS downhole sources in our field survey have a larger bandwidth than the surface-source records is that the OWS sources have a larger bandwidth than the surface sources. In another application, Mehta et al. (2008a, 2010) show that the conventional crosswell data contain much higher frequencies than the virtual crosswell data because the source-type of the virtual crosswell data is a vibroseis, whereas for conventional crosswell data, the source is a downhole high-frequency exciter. Another important reason for the relatively narrow bandwidth is that the high-frequency components of the crosswell wavefields generated by downhole sources are likely to be attenuated less than those generated by the surface sources because the former have shorter raypaths. For the source-receiver configuration we use, the raypaths for surface-source records always will be longer than the crosswell raypaths. Therefore, even if both the surface and the downhole sources were to have identical bandwidth, the bandwidth of a crosswell wavefield retrieved by interferometry would be lower than that of a wavefield generated by a downhole source because of additional attenuation along the longer raypaths. 
The bandwidth of the retrieved wavefield possibly can be improved by using surface sources with larger bandwidth. However, the fact that the crosswell SI contains lower frequencies than the conventional crosswell method suggests that the combination of these two methods would give us a larger bandwidth than that from each method alone.

The source-receiver configuration of our crosswell SI is identical to the conventional VSP survey but with two boreholes. The advantage of crosswell SI over VSP imaging is that crosswell SI does not require the velocity of the weathered near-surface layers to image deep structures. This has the potential to image the detailed structures below a complex overburden or obstacles. Furthermore, by using crosswell SI, downgoing reflections easily can be used for imaging because the downgoing multiple reflections in VSP data are transformed into downgoing primary reflections in the retrieved crosswell data.

\section{CONCLUSIONS}

We compare the application of the multidimensional deconvolution (MDD) and crosscorrelation (CC) seismic interferometry methods to numerically modeled and field data to retrieve crosswell seismic reflection wavefields. Our results demonstrate the feasibility of obtaining crosswell seismic reflection data by using widely separated boreholes without using downhole energy sources. Because MDD solutions require stabilization, we use a singular value decomposition (SVD) pseudoinverse solution to achieve MDD.

Our numerical modeling shows that because the data recorded from surface sources are correlated, the incident field matrix is not of full rank and the MDD problem therefore is ill-posed. Furthermore, the numerical modeling shows that the use of subsurface sources improves the rank of the incident field matrix, which indicates that a wider source distribution could be used to increase the amount of data that contributes to the retrieval of crosswell wavefields.

We apply the CC and the MDD methods to field data to retrieve the crosswell wavefields and then migrate the retrieved reflections. The retrieved images agree well with migrated data from a conventional crosswell seismic reflection survey and with P-wave velocities from well logs. The comparison of the retrieved reflected wavefields from the two methods with the reflected wavefields observed using borehole vibrators shows that both the MDD and CC methods retrieve upgoing reflections very well, and the MDD method retrieves downgoing reflections with better amplitude preservation than the $\mathrm{CC}$ method.

\section{ACKNOWLEDGMENTS}

We thank Recyclable-Fuel Storage Company and OYO Corporation for providing the field data used in this study. We thank Andrew Curtis, Kurang Mehta, and two anonymous reviewers for their constructive comments. The research of S. Minato was supported by

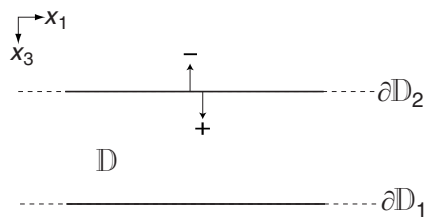

Figure A-1. Domain $\mathbb{D}$ between horizontal boundaries $\partial \mathrm{D}_{2}$ and $\partial \mathrm{D}_{1}$.
Grant-in-Aid for Japan Society for the Promotion of Science Fellows $(21 \cdot 2666)$

\section{APPENDIX A}

\section{DERIVATION OF THE MDD RELATIONSHIP FOR CROSSWELL GEOMETRY}

We derive the MDD convolution relationship for crosswell geometry (equation 7) from one-way wavefield reciprocity. Our derivation of equation 7 is almost identical to that of Wapenaar et al. (2008a) except that we consider the transmission response instead of the reflection response.

In an arbitrarily heterogeneous 3D acoustic medium, one-way wavefield reciprocity is written as

$$
\int_{\partial \mathrm{D}_{2}}\left\{\hat{p}_{A}^{+} \hat{p}_{B}^{-}-\hat{p}_{A}^{-} \hat{p}_{B}^{+}\right\} d^{2} \mathbf{x}=\int_{\partial \mathrm{D}_{1}}\left\{\hat{p}_{A}^{+} \hat{p}_{B}^{-}-\hat{p}_{A}^{-} \hat{p}_{B}^{+}\right\} d^{2} \mathbf{x},
$$

where $\mathbf{x}=\left(x_{1}, x_{2}, x_{3}\right)$ is a position vector in Cartesian coordinates, $\hat{p}$ denotes acoustic wavefield in the space-frequency domain, and the superscripts + and - denote the downgoing and upgoing acoustic waves, respectively. The symbols $\partial \mathbb{D}_{1}$ and $\partial \mathrm{D}_{2}$ denote horizontal boundaries of infinite extent above and below the domain $D$ (Figure A-1 of Appendix A). The subscripts $A$ and $B$ denote two states that have identical medium parameters inside the $3 \mathrm{D}$ domain $\mathrm{D}$ (this is the domain enclosed by the boundaries $\partial \mathrm{D}_{1}$ and $\partial \mathrm{D}_{2}$ ). Equation A-1 shows the relationship between the surface integrals of the convolution products of the decomposed wavefields for the two states $A$ and $B$. As we are interested in a crosswell geometry, we can rotate the geometry from Figure A-1 $90^{\circ}$ clockwise (Figure A-2a and A-2b). Note that we redefine the $x_{3}$-axis as the vertical coordinate in Figure A-2a and A-2b. Consequently, equation A-1 can be rewritten as

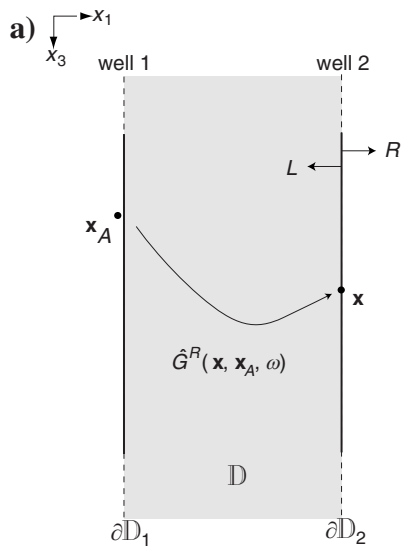

b)

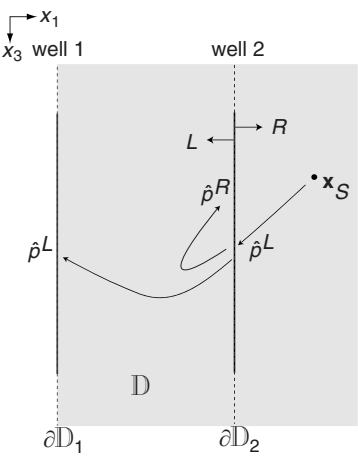

Figure A-2. (a) Desired crosswell wavefield for state $A$. The symbol $\mathrm{D}$ is a volume enclosed by the two boreholes (vertical boundaries $\partial \mathrm{D}_{1}$ and $\left.\partial \mathrm{D}_{2}\right)$ of infinite extent. The expression $\hat{G}^{R}\left(\mathbf{x}, \mathbf{x}_{A}, \omega\right)$ denotes the transmission response (or Green's function) from $\mathbf{x}_{A}$ to $\mathbf{x}$. (b) Actual wavefield for state $B$. The expressions $\hat{p}^{L}$ and $\hat{p}^{R}$ represent leftand right-propagating wavefields from the source at $\mathbf{x}_{S}$. The areas shaded in gray represent heterogeneous space. 


$$
\int_{\partial \mathrm{D}_{2}}\left\{\hat{p}_{A}^{L} \hat{p}_{B}^{R}-\hat{p}_{A}^{R} \hat{p}_{B}^{L}\right\} d^{2} \mathbf{x}=\int_{\partial \mathrm{D}_{1}}\left\{\hat{p}_{A}^{L} \hat{p}_{B}^{R}-\hat{p}_{A}^{R} \hat{p}_{B}^{L}\right\} d^{2} \mathbf{x},
$$

where the superscripts $L$ and $R$ denote the left-propagating and rightpropagating waves, respectively. The boundaries $\partial \mathrm{D}_{1}$ and $\partial \mathrm{D}_{2}$ then are vertical boundaries of infinite extent. In the following derivation, these two boundaries correspond to the borehole positions.

Let us consider two independent states $A$ and $B$ of the domain $\mathbb{D}$ that have identical medium parameters in $\mathrm{D}$. State $A$ represents the desired crosswell wavefields (Figure A-2a). For this state, we assume homogeneity for the space outside domain $\mathbb{D}$. A point source is placed at $\mathbf{x}_{A}$, which is immediately left of $\partial \mathrm{D}_{1}$. To evaluate equation A-2, we need the acoustic wavefields along $\partial \mathbb{D}_{2}$ and $\partial \mathbb{D}_{1}$. The wavefields observed at $\partial \mathrm{D}_{2}$ are right-propagating because of the homogeneity of the right half-space. Therefore, the wavefields along $\partial \mathbb{D}_{2}$ can be written to be

$$
\mathbf{x} \in \partial \mathbb{D}_{2}, \quad\left\{\begin{array}{l}
\hat{p}_{A}^{L}=0 \\
\hat{p}_{A}^{R}=\hat{G}^{R}\left(\mathbf{x}, \mathbf{x}_{A}, \omega\right) \hat{S}_{A}(\omega)
\end{array}\right.
$$

where $\hat{G}^{R}$ denotes the transmission response (or Green's function) from $\mathbf{x}_{A}$ to $\mathbf{x}$, and $\hat{S}_{A}$ denotes the source spectrum of the point source at $\mathbf{x}_{A}$. On the other hand, the wavefields along $\partial \mathbb{D}_{1}$ both are rightpropagating, caused by the point source, and left-propagating, caused by scattering inside $\mathbb{D}$. These wavefields are written

$$
\mathbf{x} \in \partial \mathbb{D}_{1}, \quad\left\{\begin{array}{l}
\hat{p}_{A}^{L}=\hat{G}^{L}\left(\mathbf{x}, \mathbf{x}_{A}, \omega\right) \hat{S}_{A}(\omega) \\
\hat{p}_{A}^{R}=\delta\left(\mathbf{x}_{\mathbf{V}}-\mathbf{x}_{\mathbf{V}, A}\right) \hat{S}_{A}(\omega)
\end{array},\right.
$$

where the subscript $\mathbf{V}$ denotes the vertical coordinate; hence, $\mathbf{x}_{\mathbf{V}}$ $=\left(x_{2}, x_{3}\right)$ and $\mathbf{x}_{\mathbf{V}, A}=\left(x_{2, A}, x_{3, A}\right)$. The latter denotes the vertical coordinate of $\mathbf{x}_{A}$.

For state $B$, we consider an actual measurement condition. The right half-space from $\partial \mathbb{D}_{2}$ can be arbitrarily inhomogeneous (Figure A-2b). A point source (a physical source) is placed at $\mathbf{x}_{S}$, which is to the right of $\partial \mathbb{D}_{2}$. The receiver arrays that are placed at $\partial \mathbb{D}_{2}$ and $\partial \mathbb{D}_{1}$ observe wavefields from this point source. In a way similar to that for state $A$, the wavefields observed at $\partial \mathrm{D}_{2}$ can be represented as

$$
\mathbf{x} \in \partial \mathbb{D}_{2}, \quad\left\{\begin{array}{l}
\hat{p}_{B}^{L}=\hat{p}^{L}\left(\mathbf{x}, \mathbf{x}_{S}, \omega\right) \\
\hat{p}_{B}^{R}=\hat{p}^{R}\left(\mathbf{x}, \mathbf{x}_{S}, \omega\right)
\end{array} .\right.
$$

Accordingly, the wavefields at $\partial \mathbb{D}_{1}$ are represented as

$$
\mathbf{x} \in \partial \mathbb{D}_{1}, \quad\left\{\begin{array}{l}
\hat{p}_{B}^{L}=\hat{p}^{L}\left(\mathbf{x}, \mathbf{x}_{S}, \omega\right) \\
\hat{p}_{B}^{R}=0
\end{array},\right.
$$

where $\hat{p}_{B}^{R}=0$ because we assume that the half-space left of $\partial \mathbb{D}_{1}$ is homogeneous. This condition seems to be unpractical for crosswell surveys in the field, but in reality it can be relaxed to a layered (horizontal or inclined) half-space, in which there are no reflected waves that propagate back to $\partial \mathrm{D}_{1}$ from the half-space left of $\partial \mathrm{D}_{1}$.

Substituting equations A-3-A-6 into equation A-2 yields the convolution relationship

$$
\hat{p}^{L}\left(\mathbf{x}_{A}, \mathbf{x}_{S}, \omega\right)=\int_{\partial \mathbb{D}_{2}} \hat{G}^{L}\left(\mathbf{x}_{A}, \mathbf{x}, \omega\right) \hat{p}^{L}\left(\mathbf{x}, \mathbf{x}_{S}, \omega\right) d^{2} \mathbf{x},
$$

where we applied source-receiver reciprocity $\left(\hat{G}^{R}\left(\mathbf{x}, \mathbf{x}_{A}, \omega\right)\right.$ $\left.=\hat{G}^{L}\left(\mathbf{x}_{A}, \mathbf{x}, \omega\right)\right)$. Note that this one-way source-receiver reciprocity relation is valid only if one used flux-normalized decomposition of the two-way wavefields into one-way wavefields (Wapenaar, 1998). Equation A-7 shows the relationship between the crosswell wavefield $\hat{G}^{L}\left(\mathbf{x}_{A}, \mathbf{x}, \omega\right)$ and the actual measurements of the responses at the borehole receiver arrays from the surface source at $\mathbf{x}_{S}$. This equation can be solved when the left-going waves $\hat{p}^{L}\left(\mathbf{x}_{A}, \mathbf{x}_{S}, \omega\right)$ and $\hat{p}^{L}\left(\mathbf{x}, \mathbf{x}_{S}, \omega\right)$ are available for a sufficient range of the source position $\mathbf{x}_{S}$. The integration of equation A-7 should be taken for the 2D surface of $\partial \mathrm{D}_{2}$. However, our borehole is one-dimensional along the $x_{3}$-direction. Therefore, in practice the integration is taken only along the vertical direction of $\partial \mathbb{D}_{2}$ with fixed borehole position $x_{2}$.

\section{REFERENCES}

Bakulin, A., and R. Calvert, 2006, The virtual source method: Theory and case study: Geophysics, 71, no. 4, SI139-SI150, doi: 10.1190/1.2216190.

Berkhout, A. J., 1982, Seismic migration: Imaging of acoustic energy by wave field extrapolation: Elsevier.

Claerbout, J. F., 1968, Synthesis of a layered medium from its acoustic transmission response: Geophysics, 33, 264-269, doi: 10.1190/1.1439927.

Curtis, A., P. Gerstoft, H. Sato, R. Snieder, and K. Wapenaar, 2006, Seismic interferometry - turning noise into signal: The Leading Edge, 25, 1082-1092, doi: 10.1190/1.2349814.

Dong, S., R. He, and G. T. Schuster, 2006, Interferometric prediction and least squares subtraction of surface waves: 76 th Annual International Meeting, SEG, Expanded Abstracts, 2783-2786.

Draganov, D., X. Campman, J. Thorbecke, A. Verdel, and K. Wapenaar, 2009, Reflection images from ambient seismic noise: Geophysics, 74, no. 5, A63-A67, doi: 10.1190/1.3193529.

Freire, S. L. M., and T. J. Ulrych, 1988, Application of singular value decomposition to vertical seismic profiling: Geophysics, 53, 778-785, doi: 10.1190/1.1442513.

Halliday, D., and A. Curtis, 2009, Seismic interferometry of scattered surface waves in attenuative media: Geophysical Journal International, 178 419-446, doi: 10.1111/j.1365-246X.2009.04153.x.

Halliday, D. F., A. Curtis, J. O. A. Robertsson, and D.-J. van Manen, 2007, Interferometric surface-wave isolation and removal: Geophysics, 72, no. 5, A69-A73.

Halliday, D. F., A. Curtis, P. Vermeer, C. Strobbia, A. Glushchenko, D.-J. van Manen, and J. O. A. Robertsson, 2010, Interferometric ground-roll removal: Attenuation of scattered surface waves in single-sensor data: Geophysics, 75, no. 2, SA15-SA25, doi: 10.1190/1.3360948.

Harris, J. M., R. C. Nolen-Hoeksema, R. T. Langan, M. Van Schaack, S. K. Lazaratos, and J. W. Rector III, 1995, High-resolution crosswell imaging of a west Texas carbonate reservoir: Part 1 -Project summary and interpretation: Geophysics, 60, 667-681, doi: 10.1190/1.1443806.

Hornby, B. E., and J. Yu, 2007, Interferometric imaging of a salt flank using walkaway VSP data: The Leading Edge, 26, 760-763, doi: 10.1190/ 1.2748493

Jiang, Z., 2006, Migration of interbed multiple reflections: 76th Annual International Meeting, SEG, Expanded Abstracts, 3501-3505.

Klema, V., and A. Laub, 1980, The singular value decomposition: Its computation and some applications: IEEE Transactions on Automatic Control, 25, 164-176, doi: 10.1109/TAC.1980.1102314.

Larose, E., L. Margerin, A. Derode, B. van Tiggelen, M. Campillo, N. Shapiro, A. Paul, L. Stehly, and M. Tanter, 2006, Correlation of random wavefields: An interdisciplinary review: Geophysics, 71, no. 4, SI11-SI21.

Lazaratos, S. K., J. W. Rector III, J. M. Harris, and M. V. Schaack, 1993, High-resolution, cross-well reflection imaging: Potential and technical difficulties: Geophysics, 58, 1270-1280, doi: 10.1190/1.1443510.

Mehta, K., A. Bakulin, D. Kiyashchenko, and J. Lopez, 2008a, Comparing virtual versus real crosswell surveys: 78th Annual International Meeting, SEG, Expanded Abstracts, 1372-1376.

Mehta, K., A. Bakulin, J. Sheiman, R. Calvert, and R. Snieder, 2007, Improving the virtual source method by wavefield separation: Geophysics, 72, no. 4, V79-V86, doi: 10.1190/1.2733020.

Mehta, K., D. Kiyashchenko, P. Jorgensen, J. Lopez, J. Ferrandis, and M. 
Costello, 2010, Virtual source method applied to crosswell and horizontal well geometries: The Leading Edge, 29, 712-723, doi: 10.1190/ 1.3447785 .

Mehta, K., R. Snieder, R. Calvert, and J. Sheiman, 2008b, Acquisition geometry requirements for generating virtual-source data: The Leading Edge, 27, 620-629, doi: 10.1190/1.2919580.

Menke, W., 1989, Geophysical data analysis: discrete inverse theory: Academic Press.

Minato, S., K. Onishi, T. Matsuoka, Y. Okajima, J. Tsuchiyama, D. Nobuoka, H. Azuma, and T. Iwamoto, 2007, Crosswell seismic survey without borehole source: 77th Annual International Meeting, SEG, Expanded Abstracts, 1357-1361.

Morozov, V. A., Z. Nashed, and A. B. Aries, 1984, Methods for solving incorrectly posed problems: Springer.

Ogura, K., T. Ohhashi, M. Osada, and M. Yoshimura, 1992, Design of a multidisk type downhole seismic source: 62nd Annual International Meeting, SEG, Expanded Abstracts, 702-705.

Oristaglio, M. L., 1985, A guide to current uses of vertical seismic profiles: Geophysics, 50, 2473-2479, doi: 10.1190/1.1441878.

Schuster, G. T., 2001, Theory of daylight/interferometric imaging: tutorial: 63rd Conference and Technical Exhibition, EAGE, Extended Abstracts, session A032.

Schuster, G. T., 2009, Seismic interferometry: Cambridge University Press.

Schuster, G. T., J. Yu, J. Sheng, and J. Rickett, 2004, Interferometric/daylight seismic imaging: Geophysical Journal International, 157, 838-852, doi: 10.1111/j.1365-246X.2004.02251.x.

Shapiro, N. M., M. Campillo, L. Stehly, and M. H. Ritzwoller, 2005, Highresolution surface-wave tomography from ambient seismic noise: Science, 307, 1615-1618, doi: 10.1126/science. 1108339 .

Slob, E., D. Draganov, and K. Wapenaar, 2007, Interferometric electromagnetic Green's functions representations using propagation invariants: Geophysical Journal International, 169, 60-80, doi: 10.1111/j.1365246X.2006.03296.x.

Snieder, R., 2004, Extracting the Green's function from the correlation of coda waves: A derivation based on stationary phase: Physical Review E: Statistical, Nonlinear, and Soft Matter Physics, 69, 046610, doi: 10.1103/ PhysRevE.69.046610

Snieder, R., K. Wapenaar, and K. Larner, 2006, Spurious multiples in seismic interferometry of primaries: Geophysics, 71, no. 4, SI111-SI124, doi: 10.1190/1.2211507.

Takahashi, T., T. Imayoshi, H. Ishikawa, and T. Takeda, 2001, Borehole seismic characterization of a heterogeneous rock: International Journal of Rock Mechanics and Mining Sciences, 38, 851-857, doi: 10.1016/S13651609(01)00050-8.
Torii, K., K. Shiraishi, K. Onishi, T. Kimura, S. Ito, T. Aizawa, K. Tsukada, and T. Matsuoka, 2006, Cross-hole tomography using seismic interferometry: 68th Conference and Technical Exhibition, EAGE, A044.

Ursin, B., and Y. Zheng, 1985, Identification of seismic reflections using singular value decomposition: Geophysical Prospecting, 33, 773-799, doi: 10.1111/j.1365-2478.1985. tb00778.x.

Vasconcelos, I., and R. Snieder, 2008a, Interferometry by deconvolution, Part 1 - theory for acoustic waves and numerical examples: Geophysics, 73, no. 3, S115-S128, doi: 10.1190/1.2904554.

Vasconcelos, I., and R. Snieder, 2008b, Interferometry by deconvolution: Part 2 - theory for elastic waves and application to drill-bit seismic imaging: Geophysics, 73, no. 3, S129-S141, doi: 10.1190/1.2904985.

Virieux, J., 1986, $P-S V$ wave propagation in heterogeneous media: Velocitystress finite-difference method: Geophysics, 51, 889-901, doi: 10.1190/ 1.1442147.

Wapenaar, K., 1998, Reciprocity properties of one-way propagators: Geophysics, 63, 1795-1798, doi: 10.1190/1.1444473.

Wapenaar, K., 2004, Retrieving the elastodynamic Green's function of an arbitrary inhomogeneous medium by cross correlation: Physical Review Letters, 93, 254301, doi: 10.1103/PhysRevLett.93.254301.

Wapenaar, K., D. Draganov, J. Thorbecke, and J. Fokkema, 2002, Theory of acoustic daylight imaging revisited: 72th Annual International Meeting, SEG, Expanded Abstracts, 2269-2272.

Wapenaar, K., and J. Fokkema, 2006, Green's function representations for seismic interferometry: Geophysics, 71, no. 4, SI33-SI46, doi: 10.1190/ 1.2213955

Wapenaar, K., E. Slob, and R. Snieder, 2008a, Seismic and electromagnetic controlled-source interferometry in dissipative media: Geophysical Prospecting, 56, 419-434, doi: 10.1111/j.1365-2478.2007.00686.x.

Wapenaar, K., J. van der Neut, and E. Ruigrok, 2008b, Passive seismic interferometry by multidimensional deconvolution: Geophysics, 73, no. 6 , A51-A56, doi: 10.1190/1.2976118.

Willis, M. E., R. Lu, X. Campman, M. N. Toksöz, Y. Zhang, and M. V. de Hoop, 2006, A novel application of time-reversed acoustics: Salt-dome flank imaging using walkaway VSP surveys: Geophysics, 71, no. 2, A7-A11, doi: 10.1190/1.2187711.

Xiao, X., M. Zhou, and G. T. Schuster, 2006, Salt-flank delineation by interferometric imaging of transmitted P- to S-waves: Geophysics, 71, no. 4, SI197-SI207, doi: 10.1190/1.2209550.

Yu, J., and G. T. Schuster, 2006, Crosscorrelogram migration of inverse vertical seismic profile data: Geophysics, 71, no. 1, S1-S11, doi: 10.1190/ 1.2159056 . 